\title{
Intermolecular Followed by Intramolecular Palladium-Catalyzed Direct Arylations for the Synthesis of $\pi$-Extended Aromatic Compounds Containing One or Two Heteroelements
}

\author{
Bilel Bouzayani, ${ }^{[a, b]}$ Ridha Ben Salem, ${ }^{*[b]}$ Jean-François Soulé ${ }^{\star[a]}$ and Henri Doucet ${ }^{*[a]}$
}

[a] B. Bouzayani, Dr. J.-F. Soulé, Dr. H. Doucet CNRS, ISCR-UMR 6226 Univ Rennes F-35000 Rennes, France E-mail: jean-francois.soule@univ-rennes1.fr, henri.doucet@univ-rennes1.fr

[b] Pr. R. Ben Salem Laboratoire de Chimie Organique LR 17ES08, Faculté des Sciences de Sfax, Université de Sfax, Route de la Soukra km 4, 3038 Sfax, Tunisia E-mail: ridhabensalem@yahoo.fr

\begin{abstract}
Herein, we report that palladium-catalyzed $\mathrm{C}-\mathrm{H}$ bond activation can overcome some of the challenges in the preparation of $\pi$-extended aromatics containing one or two heteroelements, which are important structures in organic material science. The sequential palladium-catalyzed intermolecular direct C5-arylation of heteroarenes by aryl bromides, followed by bromination of the heteroarene unit with $\mathrm{N}$-bromosuccinimide, and finally palladium-catalyzed intramolecular direct arylation allowed the synthesis of the desired phenanthro[9,10-b]thiophenes, phenanthro[9,10-b]furans, phenanthro[9,10-b]thiazoles, acenaphtho[1,2- $d]$ thiophenes and acenaphtho[1,2- $d$ ]thiazoles in only three steps.
\end{abstract}

Key topic: C-H bond functionalization

\section{Introduction}

The synthesis of $\pi$-extended polycyclic heteroaromatic hydrocarbons is currently a very hot topic in organic material science, as the introduction of heteroelements in these $\pi$-extended structures often induces a modification of their chemical and/or physical properties. For example, they are employed for the preparation of optoelectronic devices such as organic field-effect transistors (OFETs) ${ }^{[1]}$ Their unique properties are directly linked to their structures. However, the synthesis of such compounds often suffers from low functional group tolerance and/or tedious access to the key intermediates due to multi-step synthesis. Palladium-catalyzed direct functionalization through $\mathrm{C}-\mathrm{H}$ bond cleavage ${ }^{[2-4]}$ can overcome some of the challenges in the preparation of such materials. ${ }^{[5-9]}$ Indeed, the recent remarkable progress in the direct arylation of heteroaromatics or arenes has set the stage for the synthesis of complex molecules using sequential C-H bond activation/arylations. Such transformations appear as one of the most suitable methods in terms of i) number of synthetic steps, ii) functional group tolerance, iii) respect of the environment (only the formation of $\mathrm{HX}$ associated with a base as side product and no requirement to prepare and use sensitive organometallic reagents).

To our knowledge, only a few examples dealing with the synthesis of phenanthro[9,10-b]thiophenes, ${ }^{[5]}$ phenanthro[9,10-b]thiazoles, ${ }^{[6]}$ phenanthro[9,10-b]furans, ${ }^{[7]}$ acenaphtho[1,2- $\left.d\right]$ thiophenes ${ }^{[8]}$ (Scheme 1) via $\mathrm{Pd}$-catalyzed $\mathrm{C}-\mathrm{H}$ bond activation have been reported so far, and no examples of preparation of acenaphtho[1,2-d]thiazoles has been described. 

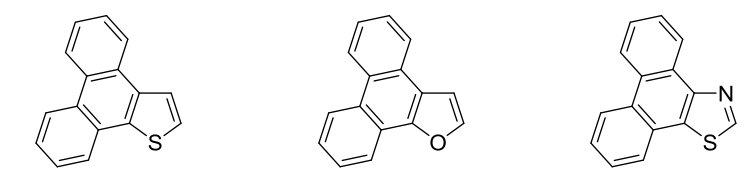

Phenanthro[9,10- $b]$ thiophene Phenanthro[9,10-b]furan Phenanthro[9,10- $d]$ thiazole

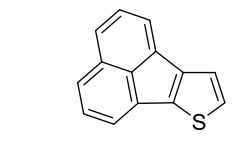

Acenaphtho[1,2-b]thiophene

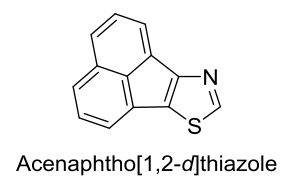

Scheme 1. Structures of some $\pi$-extended heteroaromatics.

In 2004 Höger et al. employed Pd-catalyzed C2- and C4-intramolecular direct arylations of a thiophene bearing a 2,5-dibromophenyl group at C3 position for the preparation of a phenanthro[9,10-b]thiophene derivative in $28 \%$ yield (Scheme 2, a). ${ }^{[5]}$ In 2015, Kanai, Kuninobu et al. prepared a benzo[b]phenanthro[9,10- $d$ ]thiophene from a benzothiophene substituted by an ortho-biphenyl group at C3 position via Pd-catalyzed oxidative $\mathrm{C}-\mathrm{H} / \mathrm{C}-\mathrm{H}$ coupling reaction. ${ }^{[5 b]}$ Then, in 2016 and 2017, our group reported the synthesis of phenanthro[9,10-b]thiophenes and phenanthro[9,10- $b]$ thiazoles from thiophenes and thiazoles bearing 2-bromoaryl groups at the C4 position, via C5 arylations followed by an intramolecular cyclization (Scheme 2, b). ${ }^{[5 c, 6]}$ In 2017, using a diiodobiphenyl as $\pi$-extending agent, Itami et al. produced a phenanthro[9,10-b]thiophene in one step; ${ }^{[5 \mathrm{~d}, 5 \mathrm{e}]}$ whereas, Miao et al. employed dibenzosiloles as $\pi$-extending agents (Scheme 2, c). ${ }^{[5 f]}$ A single example of synthesis of a phenanthro[9,10-b]furan via a domino Suzuki-Miyaura coupling/intramolecular direct arylation using 2bromophenylboronic acid has been described by Yoshikai et al. in 2014 (Scheme 2, d). ${ }^{[7 c]}$ By contrast, the preparation of acenaphtho[1,2- $d$ ]thiophene via $\mathrm{C}-\mathrm{H}$ bond activation remain scarce. ${ }^{[8]}$ The first example, which was reported in 1999 by Dehaen's group, deals with the ring closure of the 1,5dichloro-9,10-diarylanthracenes to produce the corresponding thiophene rubicene analogs (Scheme 2, e). ${ }^{[8]}$ 
a)<smiles>[R]c1cc(-c2ccccc2Br)c(-c2ccsc2)c(-c2ccccc2Br)c1</smiles>

b)
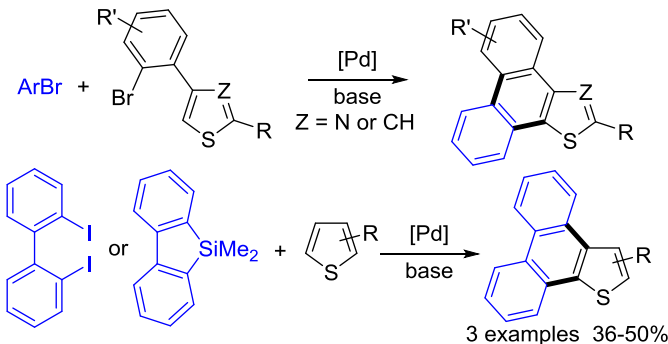

d)

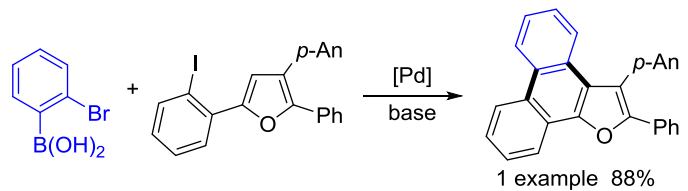

e)

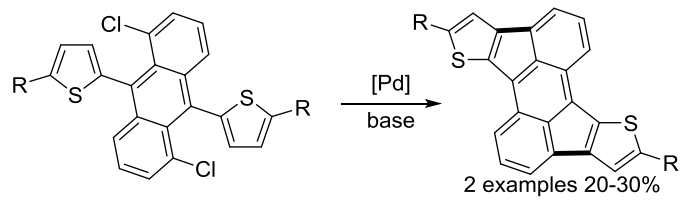

Retrosynthetic schemes of this work

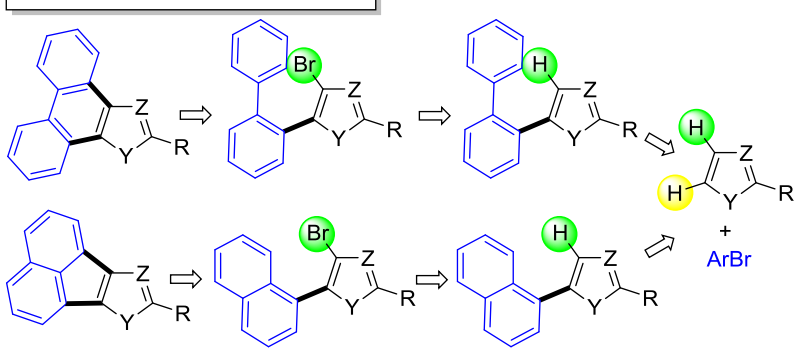

Scheme 2. Synthesis of $\mathrm{O}$ - or S-containing $\pi$-extended aromatics via a $\mathrm{C}-\mathrm{H}$ bond activation step using thiophenes, furans or thiazoles.

Herein, we report on the potential of sequential intermolecular Pd-catalyzed direct C5-arylation of heteroaromatics, regioselective C4-bromination of the heteroarene unit, and intramolecular Pdcatalyzed direct arylation for the synthesis $\pi$-extended polycyclic heteroaromatic hydrocarbons.

\section{Results and Discussion}

First, we studied the C5-arylation of a set of heteroarenes with 2-bromobiphenyl using our previously reported reaction conditions (e.g., $2 \mathrm{~mol} \% \mathrm{PdCl}\left(\mathrm{C}_{3} \mathrm{H}_{5}\right)(\mathrm{dppb})$ catalyst, KOAc as the base in DMA at $150{ }^{\circ} \mathrm{C}$ ) (Scheme 3). ${ }^{[10]} \quad$ Under these conditions, thiophene derivatives bearing acetyl, cyclopropylmethanone or ester C2-substituents gave the expected arylated products 1a-1c in 89-94\% yields. A slightly lower yield of $78 \%$ in $\mathbf{1 d}$ was obtained for the reaction of 2-acetyl-3-chlorothiophene with 2-bromobiphenyl. The reactions of 2-bromobiphenyl with a furan derivative, imidazo[1,2-a]pyridine and thiazole derivatives were also successful giving rise to the products $1 \mathrm{e}-\mathbf{1} \mathbf{h}$ in $52-96 \%$ yields. Then, we introduced a 1-naphthyl C5-substituent on thiophene and thiazole derivatives using the same reaction conditions, with the exception of the catalyst which was replaced by $\operatorname{Pd}(O A c)_{2}$, as it is known that the oxidative addition of 1 -bromonaphthalene proceeds nicely with this phosphine-free catalyst. ${ }^{[1]}$ 
In all cases, the expected products $1 \mathbf{i}$ and $1 \mathbf{k}-1 \mathrm{~m}$ were obtained in high yields. Similar yields were obtained for the reactions of 2-acetylthiophene and 2-isobutylthiazole with 1-bromo-4fluoronaphthalene affording $\mathbf{1 j}$ and $\mathbf{1 n}$ in $85 \%$ and $91 \%$ yield, respectively. 1-Bromopyrene was also employed as the aryl source using thiophene and thiazole derivatives as reaction partners. In all cases, using again $2 \mathrm{~mol} \%$ of $\mathrm{Pd}(\mathrm{OAc})_{2}$ catalyst, the expected pyren-1-yl-substituted heteroarenes 10-1s were obtained in high yields.

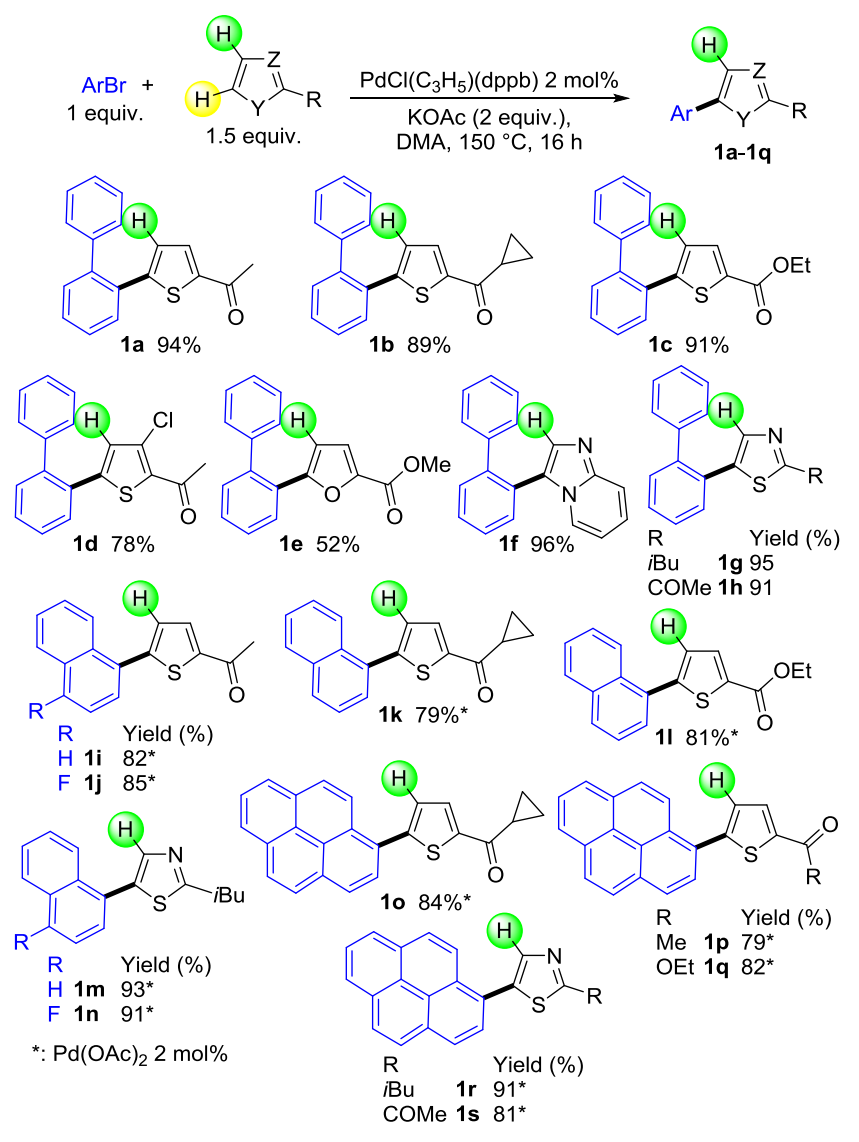

Scheme 3. Pd-catalyzed direct C5-arylations of heteroarenes with 2-bromobiphenyl, 1bromonaphthalenes and 1-bromopyrene.

Then, the reactivity of the fifteen C5-arylated heteroarenes 1a-10 in bromination reaction using $\mathrm{N}$ bromosuccinimide (NBS) as easy to handle bromine source was investigated (Scheme 4). As the biphenyl-substituted thiophenes 1a-1c contain two $\mathrm{C}-\mathrm{H}$ bonds on the thiophene ring, mixtures of brominated products might have been obtained. However, a few reports indicate that from thiophene derivatives bearing an electron-withdrawing substituent at C2-position and an alkyl or an aryl at C5position, the bromination mostly occurred at the $\mathrm{C} 4$-position; whereas, the $\mathrm{C}-\mathrm{H}$ bond at the $\mathrm{C} 3$-position remained untouched. $^{[12]}$ To our delight, the desired 4-bromo-substituted thiophene derivatives 2a-2c were obtained in high regioselectivities and in $83-86 \%$ yields. A good yield in 4-bromothiophene derivative 2d was also obtained for the bromination of 1d which contained an additional chloro-C3substituent compared to 1a. The C4-bromination of the furan ring ${ }^{[13 a]}$ of 1 e was also successful affording regioselectively $\mathbf{2} \mathbf{e}$ in $61 \%$ yield. From the imidazo[1,2-a]pyridine derivative $\mathbf{1 f}$ and NBS at 25 or $70{ }^{\circ} \mathrm{C}$, no formation of the products $2 \mathrm{f}$ was observed by GC/MS analysis of the crude mixtures, and $1 \mathrm{f}$ was recovered. To our knowledge, the bromination of the 5-membered ring of imidazo[1,2a]pyridine derivatives has been rarely described. ${ }^{[13 b]}$ This result might come from an unfavorable combination of electronic and steric properties. Conversely, the bromination of the C4-position of the 
thiazole rings ${ }^{[14]}$ of $\mathbf{1} \mathbf{g}$ and $\mathbf{1 h}$ proceeded nicely affording the expected products $\mathbf{2} \mathbf{g}$ and $\mathbf{2 h}$ in $87 \%$ and $64 \%$ yield, respectively. The naphthyl-substituted thiophenes $1 \mathbf{i}, \mathbf{1 k}$ and $\mathbf{1 l}$ treated by NBS gave the desired products $\mathbf{2} \mathbf{i}, \mathbf{2} \mathbf{k}$ and $\mathbf{2} \mathbf{I}$ in moderate yields, due to the moderate regioselectivities of these reactions. Moreover, their purification by silica chromatography afforded the expected products contaminated with unidentified side-products. The bromination of 1-(5-(4-fluoronaphthalen-1$\mathrm{yl}$ )thiophen-2-yl)ethan-1-one 1j was more selective affording the desired product $2 \mathbf{j}$ in $65 \%$ yield in pure form. The 5-naphthylthiazoles $\mathbf{1 m}$ and $1 \mathrm{n}$ were also successfully brominated, and the desired products $\mathbf{2 m}$ and $\mathbf{2 n}$ were isolated in $63 \%$ and $68 \%$ yield, respectively. It should be mentioned that the pyrene-substituted thiophenes $\mathbf{2 0 - 2 q}$ and thiazoles $2 r$ and $2 s$ could not be isolated from 1o-1s, as several unidentified products were formed in the course of these bromination reactions.

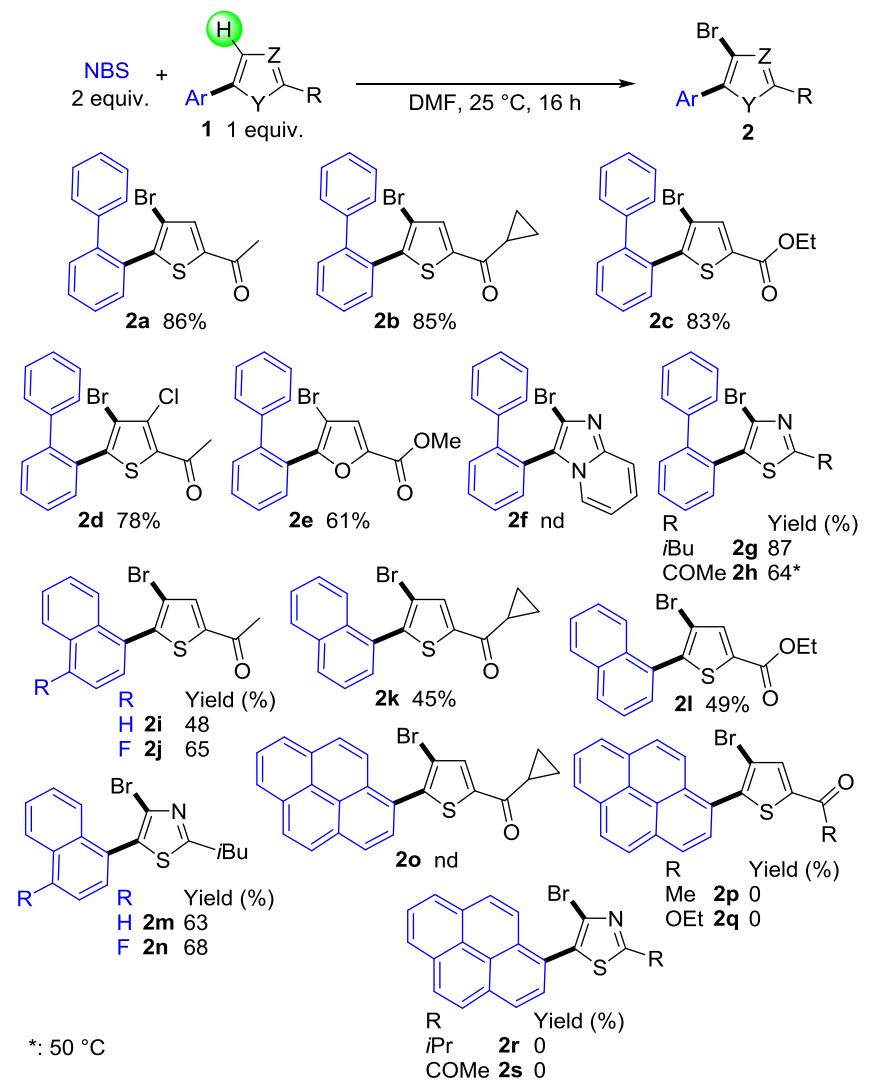

Scheme 4. C4-brominations of the C5-arylated heteroarenes 1a-1s.

Finally, we investigated the Pd-catalyzed intramolecular $\mathrm{C}-\mathrm{H}$ bond arylations of $\mathbf{2 a - 2} \mathbf{h}$ and $\mathbf{2 i - 2 \mathbf { n }}$ (Schemes 5 and 6). We tested similar conditions employed for the C5-arylations of heteroarenes of scheme 3 - namely $2 \mathrm{~mol} \%$ of $\mathrm{PdCl}\left(\mathrm{C}_{3} \mathrm{H}_{5}\right)(\mathrm{dppb})$ in DMA at $150{ }^{\circ} \mathrm{C}$ - with 2 equiv. of PivOK as the base instead of KOAc, as it was previously demonstrated that this base is very effective to promote the $\mathrm{C}-\mathrm{H}$ bond cleavage of benzene derivatives. ${ }^{[6]}$ The intramolecular arylations of $\mathbf{2 a - 2 c}$ proceeded faster than the intermolecular reactions affording the desired phenanthro[9,10-b]thiophenes $3 \mathbf{a}-\mathbf{3 c}$ in $84-87 \%$ yields. The presence of a chloro-substituent at C3-position on the thiophene ring had a deleterious influence as no formation of product $\mathbf{3} \mathbf{d}$ was detected from $\mathbf{2 d}$. Part of the starting material $\mathbf{2} \mathbf{d}$ was recovered and degradation products were also observed. The intramolecular $\mathrm{C}-\mathrm{H}$ bond arylation of the furan derivative $1 \mathbf{e}$ proceeded nicely delivering the phenanthro[9,10-b]furan $2 \mathbf{e}$ in $84 \%$ yield. A very clean cyclization reaction was also observed with the 4-bromothiazole derivative $\mathbf{2 g}$ affording the phenanthro[9,10-b]thiazole $\mathbf{3 g}$ in $90 \%$ yield. Conversely, the cyclisation of $\mathbf{2} \mathbf{h}$ was not successful, and only degradation products were obtained. 

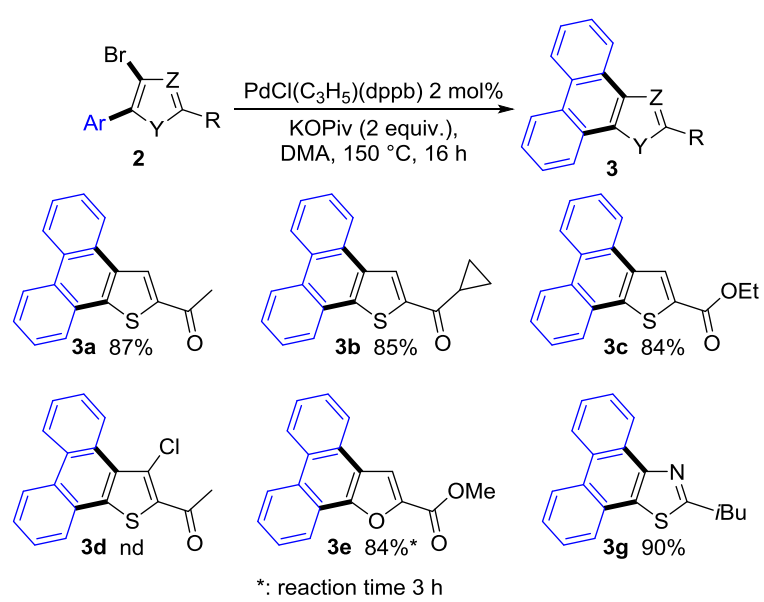

Scheme 5. Pd-catalyzed direct intramolecular arylations of bromo-substituted heteroarenes $\mathbf{2 a - 2} \mathbf{h}$.

To our knowledge, the synthesis of acenaphtho[1,2-b]thiophenes has rarely been described ${ }^{[8,15]}$ and only one example of preparation of a acenaphtho[1,2- $d$ ]thiazole has been reported. ${ }^{[16]}$ Again, we employed $2 \mathrm{~mol} \%$ of $\mathrm{PdCl}\left(\mathrm{C}_{3} \mathrm{H}_{5}\right)(\mathrm{dppb})$ with 2 equiv. of PivOK as base/ligand in DMA to promote the cyclization of the 5-naphthylthiophenes $\mathbf{2 i - 2 n}$ (Scheme 6). The desired acenaphtho[1,2-b]thiophenes $\mathbf{4 i}$, $4 \mathbf{k}$ and $\mathbf{4} \mathbf{l}$ were only obtained in $38-53 \%$ yields, from $\mathbf{2} \mathbf{i}, \mathbf{2} \mathbf{k}$ and $\mathbf{2}$ due to the formation of degradation side-products. Unexpectedly, no formation of the expected acenaphtho[1,2-b]thiophene was observed in GC/MS of the crude mixture using the fluoro-substituted 5-naphthylthiophene $2 \mathrm{j}$. In the course of this reaction, a complete conversion of $\mathbf{2} \mathbf{j}$ was observed, but only unidentified sideproducts were formed. The Pd-catalyzed annulation using 5-naphthylthiazole $\mathbf{2 m}$ was moderately successful, as the desired acenaphtho[1,2-d]thiazole $\mathbf{4 m}$ was only obtained in $21 \%$ yield. With 4 bromo-5-(4-fluoronaphthalen-1-yl)-2-isobutylthiazole $2 \mathrm{n}$, a mixture of starting material and debrominated compound 1n was obtained; whereas, the expected acenaphtho[1,2-d]thiazole was not detected. 


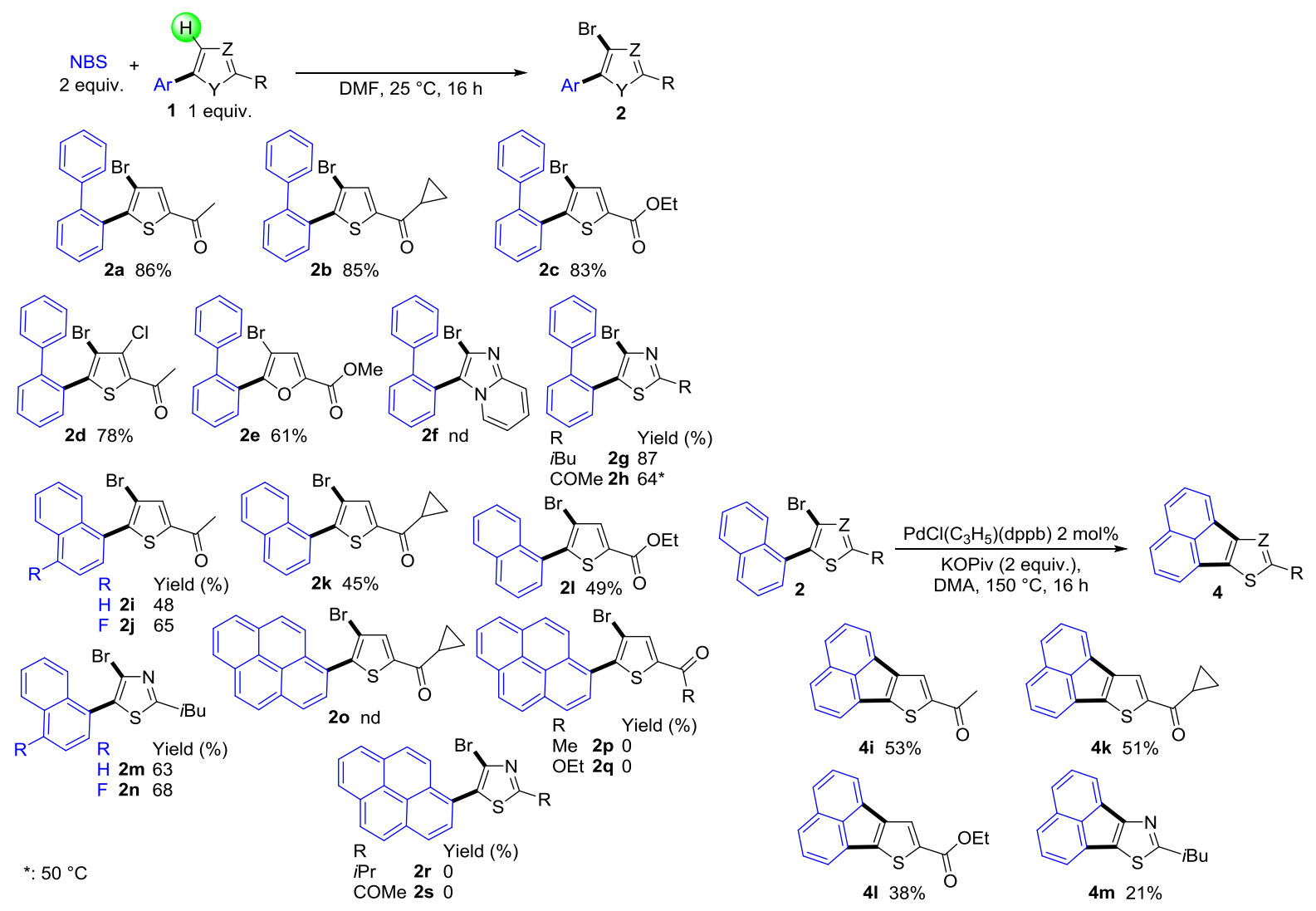

Scheme 6. Pd-catalyzed direct intramolecular arylations of the 4-bromo-substituted heteroarenes $\mathbf{2 i - 2 n}$.

\section{Conclusions}

In summary, we have investigated the potential of the intermolecular Pd-catalyzed direct C5-arylation of heteroaromatics followed by their regioselective C4-bromination and intramolecular Pd-catalyzed annulation reaction for the synthesis of $\pi$-extended aromatic compounds containing heteroelements. With most heteroaromatics, the first step proceeded in high yields using 2-bromobiphenyl, 1 bromonaphthalene or 1-bromopyrene as aryl sources and $2 \mathrm{~mol} \%$ of $\mathrm{PdCl}\left(\mathrm{C}_{3} \mathrm{H}_{5}\right)(\mathrm{dppb})$ as air-stable catalyst associated to KOAc as inexpensive base. The resulting coupling products were submitted to C4-bromination reactions of the heteroarene unit using $\mathrm{N}$-bromosuccinimide as bromination agent. Both biphenyl- and naphthyl-substituted thiophenes and thiazoles were regioselectively brominated affording the target products; whereas, pyrene-substituted heteroarenes gave complex mixtures of products. Finally, the intramolecular Pd-catalyzed annulation reaction afforded the $\pi$-extended aromatic compounds with phenanthro[9,10-b]thiophene, phenanthro[9,10-b]furan, phenanthro[9,10b]thiazole, acenaphtho[1,2- $d$ ]thiophene or acenaphtho[1,2-d]thiazole skeletons in low to high yields. Therefore, this synthetic pathway should overcome some of the challenges in the preparation of $\pi$ extended heteroaromatics containing one or two heteroelements useful in organic material science.

\section{Experimental Section}

All reactions were carried out under argon atmosphere with standard Schlenk techniques. DMA was purchased from Acros Organics and was not purified before use. ${ }^{1} \mathrm{H}$ NMR spectra were recorded on Bruker GPX (400 MHz) spectrometer. Chemical shifts $(\delta)$ were reported in parts per million relative to residual chloroform (7.28 ppm for ${ }^{1} \mathrm{H} ; 77.0 \mathrm{ppm}$ for $\left.{ }^{13} \mathrm{C}\right)$, constants were reported in Hertz. ${ }^{1} \mathrm{H}$ NMR 
assignment abbreviations were the following: singlet (s), doublet $(\mathrm{d})$, triplet (t), quartet $(\mathrm{q})$, doublet of doublets (dd), doublet of triplets (dt), and multiplet $(\mathrm{m}) \cdot{ }^{13} \mathrm{C} \mathrm{NMR}$ spectra were recorded at $100 \mathrm{MHz}$ on the same spectrometer and reported in ppm. All reagents were weighed and handled in air.

Preparation of the $\mathrm{PdCl}\left(\mathrm{C}_{3} \mathrm{H}_{5}\right)(\mathrm{dppb})$ catalyst: ${ }^{[17]}$ An oven-dried $40 \mathrm{~mL}$ Schlenk tube equipped with a magnetic stirring bar under argon atmosphere, was charged with $\left[\mathrm{Pd}\left(\mathrm{C}_{3} \mathrm{H}_{5}\right) \mathrm{Cl}_{2}(182 \mathrm{mg}, 0.5 \mathrm{mmol})\right.$ and dppb (426 mg, $1 \mathrm{mmol}$ ). $10 \mathrm{~mL}$ of anhydrous dichloromethane were added, then, the solution was stirred at room temperature for twenty minutes. The solvent was removed in vacuum. The powder was used without purification. $\left({ }^{31} \mathrm{P} 381 \mathrm{MHz}, \mathrm{CDCl}_{3}\right) \delta=19.3$ (s).

Procedure A (Direct intermolecular arylation of heteroarenes): To a $25 \mathrm{~mL}$ oven dried Schlenk tube, aryl bromide (1 mmol), heteroarene derivative $(1.5 \mathrm{mmol})$, KOAc $(0.196 \mathrm{~g}, 2 \mathrm{mmol})$, DMA (2 mL) and $\mathrm{PdCl}\left(\mathrm{C}_{3} \mathrm{H}_{5}\right)(\mathrm{dppb})(12.2 \mathrm{mg}, 0.02 \mathrm{mmol})$ or $\mathrm{Pd}(\mathrm{OAc})_{2}(4.4 \mathrm{mg}, 0.02 \mathrm{mmol})$ (see scheme) were successively added. The reaction mixture was evacuated by vacuum-argon cycles (5 times) and stirred at $150{ }^{\circ} \mathrm{C}$ (oil bath temperature) for 16 hours. After cooling the reaction at room temperature and concentration, the crude mixture was purified by silica column chromatography (diethyl ether:heptane 1:9) to afford the desired arylated products 1a-1r.

\section{1-(5-([1,1'-Biphenyl]-2-yl)thiophen-2-yl)ethan-1-one (1a)}

Following the procedure A, from 2-acetylthiophene $(0.189 \mathrm{~g}, 1.5 \mathrm{mmol})$, 2-bromobiphenyl $(0.233 \mathrm{~g}, 1$ $\mathrm{mmol})$ and $\mathrm{PdCl}\left(\mathrm{C}_{3} \mathrm{H}_{5}\right)(\mathrm{dppb})(12.2 \mathrm{mg}, 0.02 \mathrm{mmol})$, compound 1a was obtained in $94 \%$ yield $(0.261 \mathrm{~g})$ as a white solid: $\mathrm{mp} 109-111^{\circ} \mathrm{C}$.

${ }^{1} \mathrm{H}$ NMR $\left(400 \mathrm{MHz}, \mathrm{CDCl}_{3}\right) \delta(\mathrm{ppm})$ 7.61-7.56 (m, 1H), 7.49-7.40 (m, 4H), 7.36-7.31 (m, 3H), 7.29-7.25 $(\mathrm{m}, 2 \mathrm{H}), 6.65(\mathrm{~d}, J=3.9 \mathrm{~Hz}, 1 \mathrm{H}), 2.50(\mathrm{~s}, 3 \mathrm{H})$.

${ }^{13} \mathrm{C}$ NMR $\left(100 \mathrm{MHz}, \mathrm{CDCl}_{3}\right) \delta(\mathrm{ppm}) 190.5,152.1,143.7,141.2,140.9,132.6,132.2,131.1,130.5$, $129.4,129.0,128.3,128.2,127.9,127.4,26.6$.

Elemental analysis: calcd (\%) for $\mathrm{C}_{18} \mathrm{H}_{14} \mathrm{OS}$ (278.37): C 77.67, H 5.07; found: C 77.92, H 5.02.

(5-([1,1'-Biphenyl]-2-yl)thiophen-2-yl)(cyclopropyl)methanone (1b) Following the procedure A, from cyclopropyl(thiophen-2-yl)methanone $(0.228 \mathrm{~g}, 1.5 \mathrm{mmol}), 2$-bromobiphenyl $(0.233 \mathrm{~g}, 1 \mathrm{mmol})$ and $\mathrm{PdCl}\left(\mathrm{C}_{3} \mathrm{H}_{5}\right)(\mathrm{dppb})(12.2 \mathrm{mg}, 0.02 \mathrm{mmol})$, compound $\mathbf{1 b}$ was obtained in $89 \%$ yield $(0.270 \mathrm{~g})$ as a white solid: $\mathrm{mp} 128-130^{\circ} \mathrm{C}$.

${ }^{1} \mathrm{H}$ NMR $\left(400 \mathrm{MHz}, \mathrm{CDCl}_{3}\right) \delta(\mathrm{ppm}) 7.63-7.60(\mathrm{~m}, 1 \mathrm{H}), 7.59(\mathrm{~d}, J=3.9 \mathrm{~Hz}, 1 \mathrm{H}), 7.50-7.40(\mathrm{~m}, 3 \mathrm{H})$, 7.40-7.25 (m, 5H), $6.68(\mathrm{~d}, J=3.9 \mathrm{~Hz}, 1 \mathrm{H}), 2.53-2.45(\mathrm{~m}, 1 \mathrm{H}), 1.30-1.25(\mathrm{~m}, 2 \mathrm{H}), 1.05-0.99(\mathrm{~m}, 2 \mathrm{H})$.

${ }^{13} \mathrm{C}$ NMR $\left(100 \mathrm{MHz}, \mathrm{CDCl}_{3}\right) \delta(\mathrm{ppm}) 192.7,151.6,144.2,141.2,141.0,132.3,131.7,131.1,130.6$, 129.6, 128.9, 128.4, 127.8, 127.4, 17.8, 11.4 .

Elemental analysis: calcd (\%) for $\mathrm{C}_{20} \mathrm{H}_{16} \mathrm{OS}$ (304.41): C 78.91, H 5.30; found: C 79.14, H 5.39.

\section{Ethyl 5-([1,1'-biphenyl]-2-yl)thiophene-2-carboxylate (1c)}

Following the procedure A, from ethyl thiophene-2-carboxylate $(0.234 \mathrm{~g}, 1.5 \mathrm{mmol}), 2$-bromobiphenyl $(0.233 \mathrm{~g}, 1 \mathrm{mmol})$ and $\mathrm{PdCl}\left(\mathrm{C}_{3} \mathrm{H}_{5}\right)(\mathrm{dppb})(12.2 \mathrm{mg}, 0.02 \mathrm{mmol})$, compound $1 \mathrm{c}$ was obtained in $91 \%$ yield $(0.280 \mathrm{~g})$ as a yellow oil. 
${ }^{1} \mathrm{H}$ NMR $\left(400 \mathrm{MHz}, \mathrm{CDCl}_{3}\right) \delta(\mathrm{ppm})$ 7.63-7.57 (m, 2H), 7.39-7.34 (m, 3H), 7.34-7.27 (m, 5H), $6.67(\mathrm{~d}, J$ $=3.9 \mathrm{~Hz}, 1 \mathrm{H}), 4.38(\mathrm{q}, J=7.6 \mathrm{~Hz}, 2 \mathrm{H}), 1.40(\mathrm{t}, J=7.6 \mathrm{~Hz}, 3 \mathrm{H})$.

${ }^{13} \mathrm{C}$ NMR $\left(100 \mathrm{MHz}, \mathrm{CDCl}_{3}\right) \delta(\mathrm{ppm}) 162.2,150.5,141.2,140.9,133.3,132.3,131.1,130.6,129.6$, $128.8,128.3,127.9,127.8,127.4,61.1,14.5$.

Elemental analysis: calcd (\%) for $\mathrm{C}_{19} \mathrm{H}_{16} \mathrm{O}_{2} \mathrm{~S}$ (308.40): C 74.00, H 5.23; found: C 73.78, H 4.98.

\section{1-(5-([1,1'-Biphenyl]-2-yl)-3-chlorothiophen-2-yl)ethan-1-one (1d)}

Following the procedure A, from 1-(3-chlorothiophen-2-yl)ethan-1-one (0.240 g, $1.5 \mathrm{mmol})$, 2bromobiphenyl $(0.233 \mathrm{~g}, 1 \mathrm{mmol})$ and $\mathrm{PdCl}\left(\mathrm{C}_{3} \mathrm{H}_{5}\right)(\mathrm{dppb})(12.2 \mathrm{mg}, 0.02 \mathrm{mmol})$, compound 1d was obtained in $78 \%$ yield $(0.243 \mathrm{~g})$ as a yellow oil.

${ }^{1} \mathrm{H}$ NMR $\left(400 \mathrm{MHz}, \mathrm{CDCl}_{3}\right) \delta(\mathrm{ppm})$ 7.58-7.52 (m, 1H), 7.50-7.38 (m, 3H), 7.38-7.33 (m, 3H), 7.28-7.23 $(\mathrm{m}, 2 \mathrm{H}), 6.58(\mathrm{~s}, 1 \mathrm{H}), 2.61(\mathrm{~s}, 3 \mathrm{H})$.

${ }^{13} \mathrm{C}$ NMR $\left(100 \mathrm{MHz}, \mathrm{CDCl}_{3}\right) \delta(\mathrm{ppm}) 189.7,149.5,141.2,140.3,137.0,131.2,131.1,130.1,130.0$, $129.5,129.4,128.5,128.0,127.9,127.7,29.6$.

Elemental analysis: calcd (\%) for $\mathrm{C}_{18} \mathrm{H}_{13} \mathrm{ClOS}$ (312.81): C 69.11, H 4.19; found: C 69.30, H 4.28.

\section{Methyl 5-([1,1'-biphenyl]-2-yl)furan-2-carboxylate (1e)}

Following the procedure A, from methyl furan-2-carboxylate $(0.189 \mathrm{~g}, 1.5 \mathrm{mmol}), 2$-bromobiphenyl $(0.233 \mathrm{~g}, 1 \mathrm{mmol})$ and $\mathrm{PdCl}\left(\mathrm{C}_{3} \mathrm{H}_{5}\right)(\mathrm{dppb})(12.2 \mathrm{mg}, 0.02 \mathrm{mmol})$, compound $1 \mathrm{e}$ was obtained in $52 \%$ yield $(0.144 \mathrm{~g})$ as a yellow oil.

${ }^{1} \mathrm{H}$ NMR $\left(400 \mathrm{MHz}, \mathrm{CDCl}_{3}\right) \delta(\mathrm{ppm}) 7.96(\mathrm{~d}, J=7.4 \mathrm{~Hz}, 1 \mathrm{H}), 7.47-7.34(\mathrm{~m}, 5 \mathrm{H}), 7.34-7.24(\mathrm{~m}, 3 \mathrm{H}), 6.97$ $(\mathrm{d}, J=3.6 \mathrm{~Hz}, 1 \mathrm{H}), 5.56(\mathrm{~d}, J=3.6 \mathrm{~Hz}, 1 \mathrm{H}), 3.88(\mathrm{~s}, 3 \mathrm{H})$.

${ }^{13} \mathrm{C}$ NMR $\left(100 \mathrm{MHz}, \mathrm{CDCl}_{3}\right) \delta(\mathrm{ppm}) 159.2,156.5,142.9,141.4,140.4,130.8,129.1,128.6,128.4$, 127.7, 127.5, 119.5, 111.1, 51.8 .

Elemental analysis: calcd (\%) for $\mathrm{C}_{18} \mathrm{H}_{14} \mathrm{O}_{3}$ (278.31): C 77.68, H 5.07; found: C 77.52, H 4.88.

\section{3-([1,1'-Biphenyl]-2-yl)imidazo[1,2-a]pyridine (1f)}

Following the procedure A, from imidazo[1,2-a]pyridine $(0.177 \mathrm{~g}, 1.5 \mathrm{mmol})$, 2-bromobiphenyl $(0.233 \mathrm{~g}$, $1 \mathrm{mmol}$ ) and $\mathrm{PdCl}\left(\mathrm{C}_{3} \mathrm{H}_{5}\right)(\mathrm{dppb})(12.2 \mathrm{mg}, 0.02 \mathrm{mmol})$, compound $1 \mathrm{f}$ was obtained in $96 \%$ yield $(0.259$ g) as a yellow oil.

${ }^{1} \mathrm{H}$ NMR $\left(400 \mathrm{MHz}, \mathrm{CDCl}_{3}\right) \delta(\mathrm{ppm}) 7.52(\mathrm{~s}, 1 \mathrm{H}), 7.51-7.33(\mathrm{~m}, 6 \mathrm{H})$, 7.14-6.98 $(\mathrm{m}, 5 \mathrm{H})$, 6.91-6.86 (m, $1 \mathrm{H}), 6.31(\mathrm{t}, J=6.7 \mathrm{~Hz}, 1 \mathrm{H})$.

${ }^{13} \mathrm{C}$ NMR $\left(100 \mathrm{MHz}, \mathrm{CDCl}_{3}\right) \delta(\mathrm{ppm}) 145.3,141.5,140.5,133.6,131.9,130.6,129.3,128.3,127.9$, $127.8,127.2,127.1,124.9,123.8,123.4,117.5,111.5$.

Elemental analysis: calcd (\%) for $\mathrm{C}_{19} \mathrm{H}_{14} \mathrm{~N}_{2}$ (270.34): C 84.42, H 5.22; found: C 84.59, H 5.00. 
Following the procedure A, from 2-isobutylthiazole $(0.211 \mathrm{~g}, 1.5 \mathrm{mmol}), 2$-bromobiphenyl $(0.233 \mathrm{~g}, 1$ $\mathrm{mmol})$ and $\mathrm{PdCl}\left(\mathrm{C}_{3} \mathrm{H}_{5}\right)(\mathrm{dppb})(12.2 \mathrm{mg}, 0.02 \mathrm{mmol})$, compound $\mathbf{1 g}$ was obtained in $95 \%$ yield $(0.278 \mathrm{~g})$ as a colorless oil.

${ }^{1} \mathrm{H}$ NMR $\left(400 \mathrm{MHz}, \mathrm{CDCl}_{3}\right) \delta(\mathrm{ppm})$ 7.57-7.52 (m, $\left.1 \mathrm{H}\right), 7.43(\mathrm{~s}, 1 \mathrm{H}), 7.41-7.38(\mathrm{~m}, 3 \mathrm{H})$, 7.34-7.30 (m, $3 \mathrm{H}), 7.30-7.23(\mathrm{~m}, 2 \mathrm{H}), 2.78(\mathrm{~d}, J=7.6 \mathrm{~Hz}, 2 \mathrm{H}), 2.11-2.00(\mathrm{~m}, 1 \mathrm{H}), 0.97(\mathrm{~d}, J=7.6 \mathrm{~Hz}, 6 \mathrm{H})$.

${ }^{13} \mathrm{C}$ NMR $\left(100 \mathrm{MHz}, \mathrm{CDCl}_{3}\right) \delta(\mathrm{ppm}) 170.5,141.3,140.9,140.5,137.2,130.6,130.4,130.2,129.7$, 128.3, 128.2, 127.7, 127.3, 42.2, 29.8, 22.3.

Elemental analysis: calcd (\%) for $\mathrm{C}_{19} \mathrm{H}_{19} \mathrm{NS}$ (293.43): C 77.77, H 6.53; found: C 77.59, H 6.35.

\section{1-(5-([1,1'Biphenyl]-2-yl)thiazol-2-yl)ethan-1-one (1h)}

Following the procedure A, from 2-acetylthiazole $(0.190 \mathrm{~g}, 1.5 \mathrm{mmol})$, 2-bromobiphenyl $(0.233 \mathrm{~g}, 1$ $\mathrm{mmol})$ and $\mathrm{PdCl}\left(\mathrm{C}_{3} \mathrm{H}_{5}\right)(\mathrm{dppb})(12.2 \mathrm{mg}, 0.02 \mathrm{mmol})$, compound $\mathbf{1 h}$ was obtained in $91 \%$ yield $(0.254 \mathrm{~g})$ as a white solid $\mathrm{mp} 117-119^{\circ} \mathrm{C}$.

${ }^{1} \mathrm{H}$ NMR $\left(400 \mathrm{MHz}, \mathrm{CDCl}_{3}\right) \delta(\mathrm{ppm}) 7.57(\mathrm{~s}, 1 \mathrm{H}), 7.55(\mathrm{~d}, J=8.1 \mathrm{~Hz}, 1 \mathrm{H}), 7.50-7.38(\mathrm{~m}, 3 \mathrm{H})$, 7.34-7.30 $(\mathrm{m}, 3 \mathrm{H}), 7.23-7.17(\mathrm{~m}, 2 \mathrm{H}), 2.64(\mathrm{~s}, 3 \mathrm{H})$.

${ }^{13} \mathrm{C}$ NMR $\left(100 \mathrm{MHz}, \mathrm{CDCl}_{3}\right) \delta(\mathrm{ppm}) 191.7,166.1,146.1,143.3,141.7,140.2,131.0,130.7,129.5$, $129.4,129.0,128.5,127.9,127.8,25.7$.

Elemental analysis: calcd (\%) for $\mathrm{C}_{17} \mathrm{H}_{13} \mathrm{NOS}$ (279.36): C 73.09, H 4.69; found: C 73.25, H 4.47.

\section{1-(5-(Naphthalen-1-yl)thiophen-2-yl)ethan-1-one (1i)}

Following the procedure A, from 2-acetylthiophene $(0.189 \mathrm{~g}, 1.5 \mathrm{mmol}), 1$-bromonaphthalene $(0.207 \mathrm{~g}$, $1 \mathrm{mmol})$ and $\mathrm{Pd}(\mathrm{OAc})_{2}(4.4 \mathrm{mg}, 0.02 \mathrm{mmol})$, compound $1 \mathrm{i}$ was obtained in $82 \%$ yield $(0.206 \mathrm{~g})$ as a white solid: $\mathrm{mp} 113-115^{\circ} \mathrm{C}$.

${ }^{1} \mathrm{H}$ NMR $\left(400 \mathrm{MHz}, \mathrm{CDCl}_{3}\right) \delta(\mathrm{ppm}) 8.21(\mathrm{~d}, J=8.2 \mathrm{~Hz}, 1 \mathrm{H}), 7.97-7.90(\mathrm{~m}, 2 \mathrm{H}), 7.79(\mathrm{~d}, J=3.8 \mathrm{~Hz}, 1 \mathrm{H})$, 7.63-7.50 (m, 4H), $7.30(\mathrm{~d}, J=3.8 \mathrm{~Hz}, 1 \mathrm{H}), 2.65(\mathrm{~s}, 3 \mathrm{H})$.

${ }^{13} \mathrm{C}$ NMR $\left(100 \mathrm{MHz}, \mathrm{CDCl}_{3}\right) \delta(\mathrm{ppm}) 190.7,150.7,144.2,133.8,132.7,131.4,131.3,129.5,128.5$, $128.4,128.3,126.9,126.3,125.2,26.7$.

Elemental analysis: calcd (\%) for $\mathrm{C}_{16} \mathrm{H}_{12} \mathrm{OS}$ (252.33): C 76.16, H 4.79; found: C 76.34, H 4.62.

\section{1-(5-(4-Fluoronaphthalen-1-yl)thiophen-2-yl)ethan-1-one (1j)}

Following the procedure A, from 2-acetylthiophene $(0.189 \mathrm{~g}, 1.5 \mathrm{mmol}), 1$-bromo-4-fluoronaphthalene $(0.225 \mathrm{~g}, 1 \mathrm{mmol})$ and $\mathrm{Pd}(\mathrm{OAc})_{2}(4.4 \mathrm{mg}, 0.02 \mathrm{mmol})$, compound $1 \mathrm{j}$ was obtained in $85 \%$ yield $(0.229$ g) as a white solid: $\mathrm{mp} 167-169^{\circ} \mathrm{C}$.

${ }^{1} \mathrm{H}$ NMR $\left(400 \mathrm{MHz}, \mathrm{CDCl}_{3}\right) \delta(\mathrm{ppm})$ 8.23-8.14 (m, 2H), 7.79-7.75 (m, 1H), 7.64-7.56 (m, 2H), 7.55-7.49 $(\mathrm{m}, 1 \mathrm{H}), 7.28-7.17(\mathrm{~m}, 2 \mathrm{H}), 2.61(\mathrm{~s}, 3 \mathrm{H})$. 
${ }^{13} \mathrm{C}$ NMR $\left(100 \mathrm{MHz}, \mathrm{CDCl}_{3}\right) \delta(\mathrm{ppm}) 190.6,159.2(\mathrm{~d}, J=255.5 \mathrm{~Hz}), 149.9,144.3,132.7,132.6,128.5$, $128.2(\mathrm{~d}, J=8.6 \mathrm{~Hz}), 127.8,127.6(\mathrm{~d}, J=4.5 \mathrm{~Hz}), 126.6(\mathrm{~d}, J=1.9 \mathrm{~Hz}), 125.3(\mathrm{~d}, J=2.3 \mathrm{~Hz}), 124.0$ (d, $J=16.3 \mathrm{~Hz}$ ), 121.0 (d, $J=5.6 \mathrm{~Hz}), 109.0$ (d, $J=20.5 \mathrm{~Hz}), 26.7$.

Elemental analysis: calcd (\%) for $\mathrm{C}_{16} \mathrm{H}_{11} \mathrm{FOS}$ (270.32): C 71.09, H 4.10; found: C 71.25, H 4.32.

\section{Cyclopropyl(5-(naphthalen-1-yl)thiophen-2-yl)methanone (1k)}

Following the procedure A, from cyclopropyl(thiophen-2-yl)methanone $(0.228 \mathrm{~g}, 1.5 \mathrm{mmol})$, 1 bromonaphthalene $(0.207 \mathrm{~g}, 1 \mathrm{mmol})$ and $\mathrm{Pd}(\mathrm{OAc})_{2}(4.4 \mathrm{mg}, 0.02 \mathrm{mmol})$, compound $\mathbf{1 k}$ was obtained in $79 \%$ yield $(0.220 \mathrm{~g})$ as a white solid: $\mathrm{mp} 162-164{ }^{\circ} \mathrm{C}$.

${ }^{1} \mathrm{H}$ NMR $\left(400 \mathrm{MHz}, \mathrm{CDCl}_{3}\right) \delta(\mathrm{ppm}) 8.24(\mathrm{~d}, J=8.2 \mathrm{~Hz}, 1 \mathrm{H}), 7.97-7.90(\mathrm{~m}, 3 \mathrm{H}), 7.63(\mathrm{dd}, J=7.1,1.0$ $\mathrm{Hz}, 1 \mathrm{H}), 7.62-7.50(\mathrm{~m}, 3 \mathrm{H}), 7.32(\mathrm{~d}, J=3.8 \mathrm{~Hz}, 1 \mathrm{H}), 2.68-2.56(\mathrm{~m}, 1 \mathrm{H}), 1.38-1.30(\mathrm{~m}, 2 \mathrm{H}), 1.14-1.05$ $(\mathrm{m}, 2 \mathrm{H})$.

${ }^{13} \mathrm{C}$ NMR $\left(100 \mathrm{MHz}, \mathrm{CDCl}_{3}\right) \delta(\mathrm{ppm}) 192.9,150.1,144.6,133.9,131.7,131.3,129.4,128.5,128.4$, 128.3, 126.9, 126.3, 125.3, 125.2, 18.0, 11.4 .

Elemental analysis: calcd (\%) for $\mathrm{C}_{18} \mathrm{H}_{14} \mathrm{OS}$ (278.37): C 77.67, $\mathrm{H}$ 5.07; found: C 77.62, H 5.00.

\section{Ethyl 5-(naphthalen-1-yl)thiophene-2-carboxylate (1I) ${ }^{[18]}$}

Following the procedure $\mathbf{A}$, from ethyl thiophene-2-carboxylate $(0.234 \mathrm{~g}, 1.5 \mathrm{mmol})$, 1 bromonaphthalene $(0.207 \mathrm{~g}, 1 \mathrm{mmol})$ and $\mathrm{Pd}(\mathrm{OAc})_{2}(4.4 \mathrm{mg}, 0.02 \mathrm{mmol})$, compound $1 \mathrm{l}$ was obtained in $81 \%$ yield $(0.228 \mathrm{~g})$ as a colorless oil.

${ }^{1} \mathrm{H}$ NMR $\left(400 \mathrm{MHz}, \mathrm{CDCl}_{3}\right) \delta(\mathrm{ppm}) 8.25(\mathrm{~d}, J=8.2 \mathrm{~Hz}, 1 \mathrm{H}), 7.97-7.88(\mathrm{~m}, 3 \mathrm{H}), 7.61(\mathrm{dd}, J=7.1,1.1$ $\mathrm{Hz}, 1 \mathrm{H}), 7.59-7.51(\mathrm{~m}, 2 \mathrm{H}), 7.50(\mathrm{~d}, J=7.2 \mathrm{~Hz}, 1 \mathrm{H}), 7.26(\mathrm{~d}, J=3.8 \mathrm{~Hz}, 1 \mathrm{H}), 4.48(\mathrm{q}, J=7.6 \mathrm{~Hz}, 2 \mathrm{H})$, $1.47(\mathrm{t}, J=7.6 \mathrm{~Hz}, 3 \mathrm{H})$.

${ }^{13} \mathrm{C}$ NMR $\left(100 \mathrm{MHz}, \mathrm{CDCl}_{3}\right) \delta(\mathrm{ppm}) 162.3,149.0,133.9,133.7,133.6,131.5,131.4,129.4,128.6$, $128.3,128.1,126.9,126.3,125.3,125.2,61.3,14.5$.

\section{2-Isobutyl-5-(naphthalen-1-yl)thiazole (1m)}

Following the procedure $\mathbf{A}$, from 2-isobutylthiazole $(0.211 \mathrm{~g}, 1.5 \mathrm{mmol}), 1$-bromonaphthalene $(0.207 \mathrm{~g}$, $1 \mathrm{mmol})$ and $\mathrm{Pd}(\mathrm{OAc})_{2}(4.4 \mathrm{mg}, 0.02 \mathrm{mmol})$, compound $1 \mathrm{~m}$ was obtained in $93 \%$ yield $(0.248 \mathrm{~g})$ as a yellow oil.

${ }^{1} \mathrm{H}$ NMR $\left(400 \mathrm{MHz}, \mathrm{CDCl}_{3}\right) \delta(\mathrm{ppm}) 8.19(\mathrm{~d}, J=8.2 \mathrm{~Hz}, 1 \mathrm{H}), 7.91-7.81(\mathrm{~m}, 3 \mathrm{H}), 7.57-7.47(\mathrm{~m}, 3 \mathrm{H}), 7.45$ (t, $J=7.6 \mathrm{~Hz}, 1 \mathrm{H}), 2.98(\mathrm{~d}, J=7.6 \mathrm{~Hz}, 2 \mathrm{H}), 2.32-2.17(\mathrm{~m}, 1 \mathrm{H}), 1.11(\mathrm{~d}, J=7.6 \mathrm{~Hz}, 6 \mathrm{H})$.

${ }^{13} \mathrm{C}$ NMR $\left(100 \mathrm{MHz}, \mathrm{CDCl}_{3}\right) \delta(\mathrm{ppm}) 170.5,141.2,135.5,133.8,131.9,129.0(\mathrm{~m}), 128.6,128.5,126.8$, 126.2, 125.3, 125.2, 42.5, 29.9, 22.5.

Elemental analysis: calcd (\%) for $\mathrm{C}_{17} \mathrm{H}_{17} \mathrm{NS}$ (267.39): C 76.36, H 6.41; found: C 76.50, H 6.65.

\section{5-(4-Fluoronaphthalen-1-yl)-2-isobutylthiazole (1n)}


Following the procedure A, from 2-isobutylthiazole $(0.211 \mathrm{~g}, 1.5 \mathrm{mmol}), 1$-bromo-4-fluoronaphthalene $(0.225 \mathrm{~g}, 1 \mathrm{mmol})$ and $\mathrm{Pd}(\mathrm{OAc})_{2}(4.4 \mathrm{mg}, 0.02 \mathrm{mmol})$, compound $1 \mathrm{n}$ was obtained in $91 \%$ yield $(0.259$ g) as a white solid: $\mathrm{mp} 109-111^{\circ} \mathrm{C}$.

${ }^{1} \mathrm{H}$ NMR $\left(400 \mathrm{MHz}, \mathrm{CDCl}_{3}\right) \delta(\mathrm{ppm})$ 8.03-7.95 (m, 2H), $7.62(\mathrm{~s}, 1 \mathrm{H}), 7.42-7.35(\mathrm{~m}, 2 \mathrm{H}), 7.28(\mathrm{dd}, J=$ 7.9, 5.4, $1 \mathrm{H}), 6.97(\mathrm{dd}, J=10.0,7.9 \mathrm{~Hz}, 1 \mathrm{H}), 2.84(\mathrm{~d}, J=7.6 \mathrm{~Hz}, 2 \mathrm{H}), 2.20-2.00(\mathrm{~m}, 1 \mathrm{H}), 0.98(\mathrm{~d}, J=$ $7.6 \mathrm{~Hz}, 6 \mathrm{H})$.

${ }^{13} \mathrm{C} \mathrm{NMR}\left(100 \mathrm{MHz}, \mathrm{CDCl}_{3}\right) \delta(\mathrm{ppm}) 170.5,158.7$ (d, $\left.J=254.8 \mathrm{~Hz}\right), 141.2,134.6,133.1(\mathrm{~d}, J=4.9 \mathrm{~Hz})$, $128.3(\mathrm{~d}, J=8.6 \mathrm{~Hz}), 127.5,126.4(\mathrm{~d}, J=1.8 \mathrm{~Hz}), 125.2(\mathrm{~d}, J=2.6 \mathrm{~Hz}), 124.9(\mathrm{~d}, J=4.5 \mathrm{~Hz}), 123.8(\mathrm{~d}$, $J=16.3 \mathrm{~Hz}$ ), $120.8(\mathrm{~d}, J=5.7 \mathrm{~Hz}), 108.9(\mathrm{~d}, J=20.5 \mathrm{~Hz}), 42.4,29.7,22.4$.

Elemental analysis: calcd (\%) for $\mathrm{C}_{17} \mathrm{H}_{16} \mathrm{FNS}$ (285.38): C 71.55, H 5.65; found: C 71.48, H 5.36.

\section{Cyclopropyl(5-(pyren-1-yl)thiophen-2-yl)methanone (10)}

Following the procedure A, from cyclopropyl(thiophen-2-yl)methanone $(0.228 \mathrm{~g}, 1.5 \mathrm{mmol})$, 1 bromopyrene $(0.281 \mathrm{~g}, 1 \mathrm{mmol})$ and $\mathrm{Pd}(\mathrm{OAc})_{2}(4.4 \mathrm{mg}, 0.02 \mathrm{mmol})$, compound 10 was obtained in $84 \%$ yield $(0.296 \mathrm{~g})$ as a white solid: $\mathrm{mp} 193-195{ }^{\circ} \mathrm{C}$.

${ }^{1} \mathrm{H}$ NMR $\left(400 \mathrm{MHz}, \mathrm{CDCl}_{3}\right) \delta(\mathrm{ppm}) 8.49(\mathrm{~d}, J=9.3 \mathrm{~Hz}, 1 \mathrm{H}), 8.30-8.16(\mathrm{~m}, 3 \mathrm{H}), 8.15-8.02(\mathrm{~m}, 5 \mathrm{H}), 7.98$ $(\mathrm{d}, J=3.8 \mathrm{~Hz}, 1 \mathrm{H}), 7.44(\mathrm{~d}, J=3.8 \mathrm{~Hz}, 1 \mathrm{H}), 2.70-2.61(\mathrm{~m}, 1 \mathrm{H}), 1.40-1.32(\mathrm{~m}, 2 \mathrm{H}), 1.16-1.07(\mathrm{~m}, 2 \mathrm{H})$.

${ }^{13} \mathrm{C}$ NMR $\left(100 \mathrm{MHz}, \mathrm{CDCl}_{3}\right) \delta(\mathrm{ppm}) 192.9,150.8,145.1,131.8,131.7,131.4,130.8,129.0,128.9$, 128.6, 128.5, 128.3, 128.1, 127.3, 126.3, 125.7, 125.4, 125.0, 124.7, 124.4, 18.0, 11.4.

Elemental analysis: calcd (\%) for $\mathrm{C}_{24} \mathrm{H}_{16} \mathrm{OS}$ (352.45): $\mathrm{C} 81.79, \mathrm{H} 4.58$; found: $\mathrm{C} 81.90, \mathrm{H}$ 4.38.

\section{1-(5-(Pyren-1-yl)thiophen-2-yl)ethan-1-one (1p) ${ }^{[19]}$}

Following the procedure A, from 2-acetylthiophene $(0.189 \mathrm{~g}, 1.5 \mathrm{mmol}), 1$-bromopyrene $(0.281 \mathrm{~g}, 1$ $\mathrm{mmol}$ ) and $\mathrm{Pd}(\mathrm{OAc})_{2}(4.4 \mathrm{mg}, 0.02 \mathrm{mmol})$, compound $1 \mathrm{p}$ was obtained in $79 \%$ yield $(0.257 \mathrm{~g})$ as a white solid $\mathrm{mp} 116-118^{\circ} \mathrm{C}$.

${ }^{1} \mathrm{H}$ NMR $\left(400 \mathrm{MHz}, \mathrm{CDCl}_{3}\right) \delta(\mathrm{ppm}) 8.46(\mathrm{~d}, J=9.3 \mathrm{~Hz}, 1 \mathrm{H}), 8.24-8.01(\mathrm{~m}, 8 \mathrm{H}), 7.83(\mathrm{~d}, J=3.8 \mathrm{~Hz}, 1 \mathrm{H})$, $7.40(\mathrm{~d}, J=3.8 \mathrm{~Hz}, 1 \mathrm{H}), 2.68(\mathrm{~s}, 3 \mathrm{H})$.

${ }^{13} \mathrm{C}$ NMR $\left(100 \mathrm{MHz}, \mathrm{CDCl}_{3}\right) \delta(\mathrm{ppm}) 190.8,151.6,144.7,132.9,131.8,131.5,130.9,129.1,129.0$, $128.7,128.6,128.5,128.2,127.4,126.5,125.9,125.5,125.1,124.8,124.7,124.4,26.9$.

\section{Ethyl 5-(pyren-1-yl)thiophene-2-carboxylate (1q)}

Following the procedure $\mathbf{A}$, from ethyl thiophene-2-carboxylate $(0.234 \mathrm{~g}, 1.5 \mathrm{mmol}), 1$-bromopyrene $(0.281 \mathrm{~g}, 1 \mathrm{mmol})$ and $\mathrm{Pd}(\mathrm{OAc})_{2}(4.4 \mathrm{mg}, 0.02 \mathrm{mmol})$, compound $1 \mathrm{q}$ was obtained in $82 \%$ yield $(0.292$ g) as a white solid: $\mathrm{mp} 146-148^{\circ} \mathrm{C}$.

${ }^{1} \mathrm{H}$ NMR $\left(400 \mathrm{MHz}, \mathrm{CDCl}_{3}\right) \delta(\mathrm{ppm}) 8.47(\mathrm{~d}, J=9.3 \mathrm{~Hz}, 1 \mathrm{H}), 8.29-8.17(\mathrm{~m}, 3 \mathrm{H}), 8.16-8.03(\mathrm{~m}, 5 \mathrm{H}), 7.97$ $(\mathrm{d}, J=3.8 \mathrm{~Hz}, 1 \mathrm{H}), 7.38(\mathrm{~d}, J=3.8 \mathrm{~Hz}, 1 \mathrm{H}), 4.45(\mathrm{q}, J=7.6 \mathrm{~Hz}, 2 \mathrm{H}), 1.46(\mathrm{t}, J=7.6 \mathrm{~Hz}, 3 \mathrm{H})$.

${ }^{13} \mathrm{C}$ NMR $\left(100 \mathrm{MHz}, \mathrm{CDCl}_{3}\right) \delta(\mathrm{ppm}) 162.3,149.7,134.1,133.6,131.6,131.4,130.8,129.0,128.6$, $128.4,128.3,128.2,127.3,126.3,125.7,125.4,125.0,124.7,124.6,124.4,61.3,14.4$. 
Elemental analysis: calcd (\%) for $\mathrm{C}_{23} \mathrm{H}_{16} \mathrm{O}_{2} \mathrm{~S}$ (356.44): C 77.50, H 4.52; found: C 77.26, H 4.67.

2-Isobutyl-5-(pyren-1-yl)thiazole (1r)

Following the procedure A, from 2-isobutylthiazole $(0.211 \mathrm{~g}, 1.5 \mathrm{mmol})$, 1-bromopyrene $(0.281 \mathrm{~g}, 1$ $\mathrm{mmol}$ ) and $\mathrm{Pd}(\mathrm{OAc})_{2}(4.4 \mathrm{mg}, 0.02 \mathrm{mmol})$, compound $1 \mathrm{r}$ was obtained in $91 \%$ yield $(0.310 \mathrm{~g})$ as a yellow oil.

${ }^{1} \mathrm{H}$ NMR (400 MHz, $\left.\mathrm{CDCl}_{3}\right) \delta(\mathrm{ppm}) 8.38(\mathrm{~d}, J=9.2 \mathrm{~Hz}, 1 \mathrm{H}), 8.15-8.09(\mathrm{~m}, 2 \mathrm{H}), 8.07-7.94(\mathrm{~m}, 6 \mathrm{H}), 7.93$ (s, 1H), $3.05(\mathrm{~d}, J=7.6 \mathrm{~Hz}, 2 \mathrm{H}), 2.36-2.24(\mathrm{~m}, 1 \mathrm{H}), 1.17(\mathrm{~d}, J=7.6 \mathrm{~Hz}, 6 \mathrm{H})$.

${ }^{13} \mathrm{C}$ NMR $\left(100 \mathrm{MHz}, \mathrm{CDCl}_{3}\right) \delta(\mathrm{ppm}) 171.0,141.5,136.2,131.3,131.2,130.8,129.2,128.4,128.2$, 128.0, 127.2, 126.2, 126.0, 125.5, 125.2, 124.9, 124.6, 124.5, 124.3, 42.6, 30.0, 22.5.

Elemental analysis: calcd (\%) for $\mathrm{C}_{23} \mathrm{H}_{19} \mathrm{NS}$ (341.47): C 80.90, H 5.61; found: C 81.14, H 5.39.

\section{1-(5-(Pyren-1-yl)thiazol-2-yl)ethan-1-one (1s)}

Following the procedure A, from 2-acetylthiazole $(0.190 \mathrm{~g}, 1.5 \mathrm{mmol})$, 1-bromopyrene $(0.281 \mathrm{~g}, 1$ $\mathrm{mmol}$ ) and $\mathrm{Pd}(\mathrm{OAc})_{2}(4.4 \mathrm{mg}, 0.02 \mathrm{mmol})$, compound $1 \mathrm{~s}$ was obtained in $81 \%$ yield $(0.265 \mathrm{~g})$ as a yellow solid $\mathrm{mp} 98-100^{\circ} \mathrm{C}$.

${ }^{1} \mathrm{H}$ NMR $\left(400 \mathrm{MHz}, \mathrm{CDCl}_{3}\right) \delta(\mathrm{ppm}) 8.35(\mathrm{~d}, J=9.2 \mathrm{~Hz}, 1 \mathrm{H}), 8.28-8.19(\mathrm{~m}, 4 \mathrm{H}), 8.18-8.12(\mathrm{~m}, 2 \mathrm{H})$, 8.11-8.03 (m, 3H), $2.82(\mathrm{~s}, 3 \mathrm{H})$.

${ }^{13} \mathrm{C}$ NMR $\left(100 \mathrm{MHz}, \mathrm{CDCl}_{3}\right) \delta(\mathrm{ppm}) 191.8,166.9,145.3,144.1,132.1,131.3,130.7,129.2,129.0$, $128.7,128.4,127.2,126.5,126.0,125.7,124.9,124.7,124.6,124.5,123.8,25.9$.

Elemental analysis: calcd (\%) for $\mathrm{C}_{21} \mathrm{H}_{13} \mathrm{NOS}$ (327.40): $\mathrm{C} 77.04, \mathrm{H} 4.00$; found: C 76.69, H 3.68.

Procedure B (Bromination of compounds 1a-1s): To a $25 \mathrm{~mL}$ oven dried Schlenk tube, the arylated heteroarene derivatives 1a-1s (1 mmol), $N$-bromosuccinimide $(0.356 \mathrm{~g}, 2 \mathrm{mmol})$ and DMF $(2 \mathrm{~mL})$ were successively added. The reaction mixture was stirred at $25{ }^{\circ} \mathrm{C}$ for 16 hours. After concentration, the crude mixture was filtered by silica column chromatography (diethyl ether:heptane 1:4) to afford the desired brominated products which were used without further purification.

\section{1-(5-([1,1'-Biphenyl]-2-yl)-4-bromothiophen-2-yl)ethan-1-one (2a)}

Following the procedure $\mathbf{B}$, from 1-(5-([1,1'-biphenyl]-2-yl)thiophen-2-yl)ethan-1-one 1a $(0.278,1 \mathrm{mmol})$, compound $2 \mathrm{a}$ was obtained in $86 \%$ yield $(0.307 \mathrm{~g})$ as a colorless oil.

${ }^{1} \mathrm{H}$ NMR $\left(400 \mathrm{MHz}, \mathrm{CDCl}_{3}\right) \delta(\mathrm{ppm})$ 7.60-7.42 (m, 5H), 7.33-7.24 (m, 5H), $2.50(\mathrm{~s}, 3 \mathrm{H})$.

${ }^{13} \mathrm{C}$ NMR $\left(100 \mathrm{MHz}, \mathrm{CDCl}_{3}\right) \delta(\mathrm{ppm}) 189.7,147.3,143.3,142.5,140.3,134.9,131.6,130.6,130.4$, 129.9, 129.3, 128.2, 127.4, 127.3, 111.0, 26.4.

(5-([1,1'-Biphenyl]-2-yl)-4-bromothiophen-2-yl)(cyclopropyl)methanone (2b)

Following the procedure $\mathbf{B}$, from (5-([1,1'-biphenyl]-2-yl)thiophen-2-yl)(cyclopropyl)methanone 1b $(0.307,1 \mathrm{mmol})$, compound $\mathbf{2 b}$ was obtained in $85 \%$ yield $(0.325 \mathrm{~g})$ as a yellow oil. 
${ }^{1} \mathrm{H}$ NMR $\left(400 \mathrm{MHz}, \mathrm{CDCl}_{3}\right) \delta(\mathrm{ppm})$ 7.62-7.42 (m, 5H), 7.30-7.20 (m, 5H), 2.46-2.35 (m, 1H), 1.26-1.19 $(\mathrm{m}, 2 \mathrm{H}), 1.06-0.99(\mathrm{~m}, 2 \mathrm{H})$.

${ }^{13} \mathrm{C}$ NMR $\left(100 \mathrm{MHz}, \mathrm{CDCl}_{3}\right) \delta(\mathrm{ppm}) 192.0,146.7,143.7,142.5,140.4,134.1,131.7,130.6,130.5$, $129.9,129.3,128.2,127.4,127.3,111.1,17.8,11.9$.

\section{Ethyl 5-([1,1'-biphenyl]-2-yl)-4-bromothiophene-2-carboxylate (2c)}

Following the procedure $\mathbf{B}$, from ethyl 5-([1,1'-biphenyl]-2-yl)thiophene-2-carboxylate 1c $(0.308,1$ $\mathrm{mmol})$, compound $\mathbf{2 c}$ was obtained in $83 \%$ yield $(0.321 \mathrm{~g})$ as a colorless oil.

${ }^{1} \mathrm{H}$ NMR $\left(400 \mathrm{MHz}, \mathrm{CDCl}_{3}\right) \delta(\mathrm{ppm}) 7.63(\mathrm{~s}, 1 \mathrm{H}), 7.58-7.42(\mathrm{~m}, 4 \mathrm{H}), 7.35-7.25(\mathrm{~m}, 5 \mathrm{H}), 4.35(\mathrm{q}, J=7.6$ $\mathrm{Hz}, 2 \mathrm{H}), 1.38(\mathrm{t}, J=7.6 \mathrm{~Hz}, 3 \mathrm{H})$.

${ }^{13} \mathrm{C}$ NMR $\left(100 \mathrm{MHz}, \mathrm{CDCl}_{3}\right) \delta(\mathrm{ppm}) 161.2,145.5,142.6,140.4,135.6,133.6,131.9,130.6,130.4$, $129.9,129.3,128.2,127.4,127.3,111.0,61.6,14.4$.

\section{1-(5-([1,1'-Biphenyl]-2-yl)-4-bromo-3-chlorothiophen-2-yl)ethan-1-one (2d)}

Following the procedure $\mathbf{B}$, from 1-(5-([1,1'-biphenyl]-2-yl)-3-chlorothiophen-2-yl)ethan-1-one 1d $(0.313$, $1 \mathrm{mmol})$, compound $\mathbf{2 d}$ was obtained in $78 \%$ yield $(0.305 \mathrm{~g})$ as a white solid: $\mathrm{mp} 151-153^{\circ} \mathrm{C}$.

${ }^{1} \mathrm{H}$ NMR $\left(400 \mathrm{MHz}, \mathrm{CDCl}_{3}\right) \delta(\mathrm{ppm})$ 7.60-7.42 (m, 4H), 7.33-7.28 (m, 3H), 7.27-7.21 (m, 2H), $2.67(\mathrm{~s}$, $3 \mathrm{H})$.

${ }^{13} \mathrm{C}$ NMR $\left(100 \mathrm{MHz}, \mathrm{CDCl}_{3}\right) \delta(\mathrm{ppm}) 189.3,145.8,142.4,140.0,137.3,131.4,130.7,130.5,129.2$, 129.0, 128.3, 127.5, 127.4, 114.4, 29.7.

\section{Methyl 5-([1,1'-biphenyl]-2-yl)-4-bromofuran-2-carboxylate (2e)}

Following the procedure $\mathbf{B}$, from methyl 5-([1,1'-biphenyl]-2-yl)furan-2-carboxylate $1 \mathrm{e}(0.278,1 \mathrm{mmol})$, compound $2 \mathbf{e}$ was obtained in $61 \%$ yield $(0.218 \mathrm{~g})$ as a white solid: $\mathrm{mp} 96-98^{\circ} \mathrm{C}$.

${ }^{1} \mathrm{H}$ NMR $\left(400 \mathrm{MHz}, \mathrm{CDCl}_{3}\right) \delta(\mathrm{ppm}) 7.62(\mathrm{~d}, J=8.0 \mathrm{~Hz}, 1 \mathrm{H}), 7.57-7.47(\mathrm{~m}, 2 \mathrm{H}), 7.44(\mathrm{t}, J=7.8 \mathrm{~Hz}, 1 \mathrm{H})$, 7.34-7.26 (m, 3H), 7.24-7.18 (m, 2H), $7.14(\mathrm{~s}, 1 \mathrm{H}), 3.79(\mathrm{~s}, 3 \mathrm{H})$.

${ }^{13} \mathrm{C}$ NMR $\left(100 \mathrm{MHz}, \mathrm{CDCl}_{3}\right) \delta(\mathrm{ppm}) 158.2,154.7,143.4,142.7,140.6,130.5,130.4,128.7,128.2$, 127.3, 127.2, 126.7, 121.7, 100.1, 52.0.

\section{5-([1,1'-Biphenyl]-2-yl)-4-bromo-2-isobutylthiazole (2g)}

Following the procedure $\mathbf{B}$, from 5-([1,1'-biphenyl]-2-yl)-2-isobutylthiazole $\mathbf{1 g}(0.293,1 \mathrm{mmol})$, compound $\mathbf{2 g}$ was obtained in $87 \%$ yield $(0.323 \mathrm{~g})$ as a colorless oil.

${ }^{1} \mathrm{H}$ NMR $\left(400 \mathrm{MHz}, \mathrm{CDCl}_{3}\right) \delta(\mathrm{ppm}) 7.59-7.40(\mathrm{~m}, 4 \mathrm{H}), 7.33-7.21(\mathrm{~m}, 5 \mathrm{H}), 2.76(\mathrm{~d}, J=7.6 \mathrm{~Hz}, 2 \mathrm{H})$, 2.09-1.98 (m, 1H), $0.94(\mathrm{~d}, J=7.6 \mathrm{~Hz}, 6 \mathrm{H})$.

${ }^{13} \mathrm{C}$ NMR $\left(100 \mathrm{MHz}, \mathrm{CDCl}_{3}\right) \delta(\mathrm{ppm}) 170.7,142.8,140.4,133.8,132.1,131.9,130.3,129.4,128.8$, $128.1,127.3,124.9,123.8,42.4,29.7,22.2$. 
Following the procedure $\mathbf{B}$, from 1-(5-([1,1'biphenyl]-2-yl)thiazol-2-yl)ethan-1-one $\mathbf{1 h}(0.279,1 \mathrm{mmol})$, compound $\mathbf{2 h}$ was obtained in $64 \%$ yield $(0.229 \mathrm{~g})$ as a white solid: $\mathrm{mp} 107-109^{\circ} \mathrm{C}$.

${ }^{1} \mathrm{H}$ NMR $\left(400 \mathrm{MHz}, \mathrm{CDCl}_{3}\right) \delta(\mathrm{ppm})$ 7.63-7.55 (m, 1H), 7.54-7.45 (m, 3H), 7.32-7.28 (m, 3H), 7.22-7.18 $(\mathrm{m}, 2 \mathrm{H}), 2.68(\mathrm{~s}, 3 \mathrm{H})$.

${ }^{13} \mathrm{C}$ NMR $\left(100 \mathrm{MHz}, \mathrm{CDCl}_{3}\right) \delta(\mathrm{ppm}) 190.7,165.4,142.7,142.1,139.8,131.5,130.6,130.2,129.3$, $128.3,127.8,127.6,127.5,126.7,25.6$.

\section{1-(4-Bromo-5-(naphthalen-1-yl)thiophen-2-yl)ethan-1-one (2i)}

Following the procedure $\mathbf{B}$, from 1-(5-(naphthalen-1-yl)thiophen-2-yl)ethan-1-one 1i $(0.252,1 \mathrm{mmol}$ ), compound $2 \mathbf{i}$ was obtained in $48 \%$ yield in an impure form ( $85 \%$ purity, $0.187 \mathrm{~g}$ ) as a yellow solid: $\mathrm{mp}$ $116-118^{\circ} \mathrm{C}$.

${ }^{1} \mathrm{H}$ NMR $\left(400 \mathrm{MHz}, \mathrm{CDCl}_{3}\right) \delta(\mathrm{ppm}) 7.97(\mathrm{~d}, J=6.8 \mathrm{~Hz}, 1 \mathrm{H}), 7.93(\mathrm{~d}, J=7.2 \mathrm{~Hz}, 1 \mathrm{H}), 7.77-7.65(\mathrm{~m}, 2 \mathrm{H})$, 7.55-7.44 (m, 4H), $2.64(\mathrm{~s}, 3 \mathrm{H})$.

${ }^{13} \mathrm{C}$ NMR $\left(100 \mathrm{MHz}, \mathrm{CDCl}_{3}\right) \delta(\mathrm{ppm}) 190.6,149.5,144.6,132.6,132.4,132.2,129.4,128.8,128.3$, $127.8,127.7,127.6,125.9,124.4,26.8$.

\section{1-(4-Bromo-5-(4-fluoronaphthalen-1-yl)thiophen-2-yl)ethan-1-one (2j)}

Following the procedure $\mathbf{B}$, from 1-(5-(4-fluoronaphthalen-1-yl)thiophen-2-yl)ethan-1-one 1j $(0.270,1$ $\mathrm{mmol})$, compound $2 \mathrm{j}$ was obtained in $65 \%$ yield $(0.226 \mathrm{~g})$ as a white solid: $\mathrm{mp} 92-94{ }^{\circ} \mathrm{C}$.

${ }^{1} \mathrm{H}$ NMR $\left(400 \mathrm{MHz}, \mathrm{CDCl}_{3}\right) \delta(\mathrm{ppm}) 8.19(\mathrm{~d}, J=7.8 \mathrm{~Hz}, 1 \mathrm{H}), 7.75(\mathrm{~s}, 1 \mathrm{H}), 7.72(\mathrm{~d}, J=8.0 \mathrm{~Hz}, 1 \mathrm{H})$, 7.65-7.57 (m, 2H), 7.48 (dd, $J=7.9,5.3 \mathrm{~Hz}, 1 \mathrm{H}), 7.24(\mathrm{dd}, J=10.0,8.0 \mathrm{~Hz}, 1 \mathrm{H}), 2.61(\mathrm{~s}, 3 \mathrm{H})$.

${ }^{13} \mathrm{C}$ NMR $\left(100 \mathrm{MHz}, \mathrm{CDCl}_{3}\right) \delta(\mathrm{ppm}) 189.7,159.8(\mathrm{~d}, J=255.7 \mathrm{~Hz}), 144.6,143.7,135.0,132.8(\mathrm{~d}, J=$ $5.2 \mathrm{~Hz}$ ), 129.2 (d, J = 8.9 Hz), 127.9, 126.7 (d, J=1.9 Hz), 125.7 (d, J=2.6 Hz), 125.4 (d, J = 4.5 Hz), $123.8(\mathrm{~d}, J=16.5 \mathrm{~Hz}), 121.1$ (d, $J=5.6 \mathrm{~Hz}), 112.0,109.0$ (d, $J=20.6 \mathrm{~Hz}), 26.5$.

\section{(4-Bromo-5-(naphthalen-1-yl)thiophen-2-yl)(cyclopropyl)methanone (2k)}

Following the procedure $\mathbf{B}$, from cyclopropyl(5-(naphthalen-1-yl)thiophen-2-yl)methanone $\mathbf{1 k}(0.278,1$ $\mathrm{mmol}$ ), compound $\mathbf{2 k}$ was obtained in $45 \%$ yield as a colorless oil in an impure form (60\% purity, 0.269 g).

${ }^{1} \mathrm{H}$ NMR $\left(400 \mathrm{MHz}, \mathrm{CDCl}_{3}\right) \delta(\mathrm{ppm})$ 8.00-7.65 (m, 4H), 7.58-7.47 (m, 4H), 2.64-2.51 (m, 1H), 1.38-1.30 $(\mathrm{m}, 2 \mathrm{H}), 1.14-1.05(\mathrm{~m}, 2 \mathrm{H})$.

\section{Ethyl 4-bromo-5-(naphthalen-1-yl)thiophene-2-carboxylate (2l)}

Following the procedure $\mathbf{B}$, from ethyl 5-(naphthalen-1-yl)thiophene-2-carboxylate $1 \mathbf{1}(0.282,1 \mathrm{mmol})$, compound 2 l was obtained in $49 \%$ yield as a colorless oil in an impure form ( $75 \%$ purity, $0.236 \mathrm{~g}$ ).

${ }^{1} \mathrm{H}$ NMR $\left(400 \mathrm{MHz}, \mathrm{CDCl}_{3}\right) \delta(\mathrm{ppm}) 7.97(\mathrm{~d}, J=6.8 \mathrm{~Hz}, 1 \mathrm{H}), 7.93(\mathrm{~d}, J=7.2 \mathrm{~Hz}, 1 \mathrm{H}), 7.85-7.73(\mathrm{~m}, 2 \mathrm{H})$, 7.57-7.47 (m, 4H), $4.46(\mathrm{q}, J=7.6 \mathrm{~Hz}, 2 \mathrm{H}), 1.47(\mathrm{t}, J=7.6 \mathrm{~Hz}, 3 \mathrm{H})$.

\section{4-Bromo-2-isobutyl-5-(naphthalen-1-yl)thiazole (2m)}


Following the procedure B, from 2-isobutyl-5-(naphthalen-1-yl)thiazole $1 \mathrm{~m}(0.267,1 \mathrm{mmol})$, compound $2 \mathrm{~m}$ was obtained in $63 \%$ yield $(0.218 \mathrm{~g})$ as a white solid: $\mathrm{mp} 128-130{ }^{\circ} \mathrm{C}$.

${ }^{1} \mathrm{H}$ NMR $\left(400 \mathrm{MHz}, \mathrm{CDCl}_{3}\right) \delta(\mathrm{ppm})$ 8.00-7.91 (m, 2H), 7.83-7.76 (m, 1H), 7.60-7.50 (m, 4H), $2.97(\mathrm{~d}, J$ $=7.6 \mathrm{~Hz}, 2 \mathrm{H}), 2.31-2.17(\mathrm{~m}, 1 \mathrm{H}), 1.12(\mathrm{~d}, J=7.6 \mathrm{~Hz}, 6 \mathrm{H})$.

${ }^{13} \mathrm{C}$ NMR $\left(100 \mathrm{MHz}, \mathrm{CDCl}_{3}\right) \delta(\mathrm{ppm}) 171.1,133.7,132.0,130.2,129.9,129.7,128.5,127.6,126.9$, 126.3, 125.6, 125.2, 124.5, 42.7, 29.8, 22.4.

\section{4-Bromo-5-(4-fluoronaphthalen-1-yl)-2-isobutylthiazole (2n)}

Following the procedure $\mathbf{B}$, from 5-(4-fluoronaphthalen-1-yl)-2-isobutylthiazole $1 \mathrm{n}(0.285,1 \mathrm{mmol})$, compound $2 \mathrm{n}$ was obtained in $68 \%$ yield $(0.247 \mathrm{~g})$ as a white solid: $\mathrm{mp} 99-101^{\circ} \mathrm{C}$.

${ }^{1} \mathrm{H}$ NMR $\left(400 \mathrm{MHz}, \mathrm{CDCl}_{3}\right) \delta(\mathrm{ppm}) 8.18(\mathrm{~d}, J=7.8 \mathrm{~Hz}, 1 \mathrm{H}), 7.73(\mathrm{~d}, J=8.0 \mathrm{~Hz}, 1 \mathrm{H}), 7.62-7.53(\mathrm{~m}, 2 \mathrm{H})$, $7.47(\mathrm{dd}, J=7.9,5.3 \mathrm{~Hz}, 1 \mathrm{H}), 7.20(\mathrm{dd}, J=10.0,8.0 \mathrm{~Hz}, 1 \mathrm{H}), 2.93(\mathrm{~d}, J=7.6 \mathrm{~Hz}, 2 \mathrm{H}), 2.26-2.24(\mathrm{~m}$, $1 \mathrm{H}), 1.07$ (d, $J=7.6 \mathrm{~Hz}, 6 \mathrm{H})$.

${ }^{13} \mathrm{C}$ NMR $\left(100 \mathrm{MHz}, \mathrm{CDCl}_{3}\right) \delta(\mathrm{ppm}) 171.2,159.5(\mathrm{~d}, J=255.3 \mathrm{~Hz}), 133.4(\mathrm{~d}, J=5.2 \mathrm{~Hz}), 129.6(\mathrm{~d}, J=$ 8.9 Hz), 129.4, 127.8, 126.6 (d, $J=1.8 \mathrm{~Hz}), 125.6$ (d, $J=2.5 \mathrm{~Hz}), 124.8,123.9$ (d, $J=16.5 \mathrm{~Hz}), 123.5$ (d, $J=4.5 \mathrm{~Hz}), 121.0$ (d, $J=5.5 \mathrm{~Hz}), 109.1(\mathrm{~d}, J=20.6 \mathrm{~Hz}), 42.7,29.8,22.4$.

Procedure C (Direct intramolecular arylation of heteroarenes): To a $25 \mathrm{~mL}$ oven dried Schlenk tube, heteroaryl bromide $(0.5 \mathrm{mmol})$, KOPiv $(0.140 \mathrm{~g}, 1 \mathrm{mmol})$, DMA $(2 \mathrm{~mL})$ and $\mathrm{PdCl}\left(\mathrm{C}_{3} \mathrm{H}_{5}\right)(\mathrm{dppb})(6.1$ $\mathrm{mg}, 0.01 \mathrm{mmol})$ were successively added. The reaction mixture was evacuated by vacuum-argon cycles (5 times) and stirred at $150^{\circ} \mathrm{C}$ (oil bath temperature) for 3 or 16 hours (see scheme 5). After cooling the reaction at room temperature and concentration, the crude mixture was purified by silica column chromatography (diethyl ether:heptane 2:3) to afford the desired products.

\section{1-(Phenanthro[9,10-b]thiophen-2-yl)ethan-1-one (3a)}

Following the procedure $\mathbf{C}$, from 1-(5-([1,1'-biphenyl]-2-yl)-4-bromothiophen-2-yl)ethan-1-one $\mathbf{2 a}(0.179$ $\mathrm{g}, 0.5 \mathrm{mmol})$, compound $3 \mathrm{a}$ was obtained in $87 \%$ yield $(0.120 \mathrm{~g})$ as a yellow solid: $\mathrm{mp} 218-220^{\circ} \mathrm{C}$.

${ }^{1} \mathrm{H}$ NMR $\left(400 \mathrm{MHz}, \mathrm{CDCl}_{3}\right) \delta(\mathrm{ppm})$ 8.70-8.62 (m, 2H), $8.47(\mathrm{~s}, 1 \mathrm{H}), 8.31-8.25(\mathrm{~m}, 1 \mathrm{H}), 8.15(\mathrm{~d}, J=7.8$ $\mathrm{Hz}, 1 \mathrm{H}), 7.76-7.61(\mathrm{~m}, 4 \mathrm{H}), 2.75(\mathrm{~s}, 3 \mathrm{H})$.

${ }^{13} \mathrm{C}$ NMR $\left(100 \mathrm{MHz}, \mathrm{CDCl}_{3}\right) \delta(\mathrm{ppm}) 191.4,142.4,142.2,134.9,129.9,129.2,128.6,128.2,128.1$, $127.6(\mathrm{~m}), 127.5,126.7,124.9,123.9,123.7,123.6,26.9$.

Elemental analysis: calcd (\%) for $\mathrm{C}_{18} \mathrm{H}_{12} \mathrm{OS}$ (276.35): C 78.23, H 4.38; found: C 78.39, H 4.57.

\section{Cyclopropyl(phenanthro[9,10-b]thiophen-2-yl)methanone (3b)}

Following the procedure $\mathbf{C}$, from (5-([1,1'-biphenyl]-2-yl)-4-bromothiophen-2-yl)(cyclopropyl)methanone 2b $(0.192 \mathrm{~g}, 0.5 \mathrm{mmol})$, compound $\mathbf{3 b}$ was obtained in $85 \%$ yield $(0.128 \mathrm{~g})$ as a yellow solid: $\mathrm{mp} 247-$ $249^{\circ} \mathrm{C}$.

${ }^{1} \mathrm{H}$ NMR $\left(400 \mathrm{MHz}, \mathrm{CDCl}_{3}\right) \delta(\mathrm{ppm}) 8.68-8.60(\mathrm{~m}, 3 \mathrm{H}), 8.31(\mathrm{~d}, J=6.4 \mathrm{~Hz}, 1 \mathrm{H}), 8.17(\mathrm{~d}, J=7.8 \mathrm{~Hz}, 1 \mathrm{H})$, 7.70-7.62 (m, $4 \mathrm{H}), 2.81-2.72(\mathrm{~m}, 1 \mathrm{H}), 1.40-1.30(\mathrm{~m}, 2 \mathrm{H}), 1.20-1.10(\mathrm{~m}, 2 \mathrm{H})$. 
${ }^{13} \mathrm{C}$ NMR $\left(100 \mathrm{MHz}, \mathrm{CDCl}_{3}\right) \delta(\mathrm{ppm}) 193.5,142.9,141.8,135.0,129.8,129.2,128.8,128.0,127.7$, $127.6,127.5,127.2,126.7,124.8,124.0,123.7,123.6,18.2,11.8$.

Elemental analysis: calcd (\%) for $\mathrm{C}_{20} \mathrm{H}_{14} \mathrm{OS}$ (302.39): C 79.44, H 4.67; found: C 79.32, H 4.79.

\section{Ethyl phenanthro[9,10-b]thiophene-2-carboxylate (3c)}

Following the procedure $\mathbf{C}$, from ethyl 5-([1,1'-biphenyl]-2-yl)-4-bromothiophene-2-carboxylate 2c $(0.193 \mathrm{~g}, 0.5 \mathrm{mmol})$, compound $3 \mathrm{c}$ was obtained in $84 \%$ yield $(0.128 \mathrm{~g})$ as a white solid: $\mathrm{mp} 190-$ $192^{\circ} \mathrm{C}$.

${ }^{1} \mathrm{H}$ NMR $\left(400 \mathrm{MHz}, \mathrm{CDCl}_{3}\right) \delta(\mathrm{ppm}) 8.67(\mathrm{~d}, J=8.6 \mathrm{~Hz}, 2 \mathrm{H}), 8.64(\mathrm{~s}, 1 \mathrm{H}), 8.34-8.30(\mathrm{~m}, 1 \mathrm{H}), 8.15(\mathrm{~d}, J$ $=7.8 \mathrm{~Hz}, 1 \mathrm{H}), 7.74-7.61(\mathrm{~m}, 4 \mathrm{H}), 4.49(\mathrm{q}, J=7.6 \mathrm{~Hz}, 2 \mathrm{H}), 1.50(\mathrm{t}, J=7.6 \mathrm{~Hz}, 3 \mathrm{H})$.

${ }^{13} \mathrm{C}$ NMR $\left(100 \mathrm{MHz}, \mathrm{CDCl}_{3}\right) \delta(\mathrm{ppm}) 162.7,141.1,134.7,132.2,129.7,129.2,129.1,128.6,127.8$, 127.7, 127.5, 126.6, 124.6, 124.2, 123.7, 123.5, 61.6, 14.4.

Elemental analysis: calcd (\%) for $\mathrm{C}_{19} \mathrm{H}_{14} \mathrm{O}_{2} \mathrm{~S}$ (306.38): C 74.49, $\mathrm{H} \mathrm{4.61;} \mathrm{found:} \mathrm{C} \mathrm{74.67,} \mathrm{H} \mathrm{4.90.}$

\section{Methyl phenanthro[9,10-b]furan-2-carboxylate (3e)}

Following the procedure $\mathbf{C}$, from methyl 5-([1,1'-biphenyl]-2-yl)-4-bromofuran-2-carboxylate $\mathbf{2 e}(0.178 \mathrm{~g}$, $0.5 \mathrm{mmol})$, compound $3 \mathrm{e}$ was obtained in $84 \%$ yield $(0.116 \mathrm{~g})$ as a white solid: $\mathrm{mp} 156-158{ }^{\circ} \mathrm{C}$.

${ }^{1} \mathrm{H}$ NMR $\left(400 \mathrm{MHz}, \mathrm{CDCl}_{3}\right) \delta(\mathrm{ppm}) 8.62(\mathrm{~d}, J=6.4 \mathrm{~Hz}, 2 \mathrm{H}), 8.44-8.40(\mathrm{~m}, 1 \mathrm{H}), 8.06(\mathrm{~d}, J=7.0 \mathrm{~Hz}, 1 \mathrm{H})$, $7.93(\mathrm{~s}, 1 \mathrm{H}), 7.69-7.59(\mathrm{~m}, 4 \mathrm{H}), 4.02(\mathrm{~s}, 3 \mathrm{H})$.

${ }^{13} \mathrm{C}$ NMR $\left(100 \mathrm{MHz}, \mathrm{CDCl}_{3}\right) \delta(\mathrm{ppm}) 159.7,151.3,144.6,130.6,128.4,127.5,127.4,127.3,126.8$, 126.0, 123.9, 123.6, 123.4, 121.8, 121.6, 120.5, 113.7, 52.2.

Elemental analysis: calcd (\%) for $\mathrm{C}_{18} \mathrm{H}_{12} \mathrm{O}_{3}$ (276.29): C 78.25, H 4.38; found: C 78.39, H 4.61.

\section{2-Isobutylphenanthro[9,10-d]thiazole (3g)}

Following the procedure C, from 5-([1,1'-biphenyl]-2-yl)-4-bromo-2-isobutylthiazole $2 \mathrm{~g}$ (0.186 g, 0.5 $\mathrm{mmol})$, compound $\mathbf{3 g}$ was obtained in $90 \%$ yield $(0.131 \mathrm{~g})$ as a yellow solid: $\mathrm{mp} 118-120^{\circ} \mathrm{C}$.

${ }^{1} \mathrm{H}$ NMR $\left(400 \mathrm{MHz}, \mathrm{CDCl}_{3}\right) \delta(\mathrm{ppm}) 8.93(\mathrm{~d}, J=7.6 \mathrm{~Hz}, 1 \mathrm{H}), 8.62-8.55(\mathrm{~m}, 2 \mathrm{H}), 7.88-7.84(\mathrm{~m}, 1 \mathrm{H}), 7.77$ $(\mathrm{t}, J=7.2 \mathrm{~Hz}, 1 \mathrm{H}), 7.67(\mathrm{t}, J=7.6 \mathrm{~Hz}, 1 \mathrm{H}), 7.58-7.54(\mathrm{~m}, 2 \mathrm{H}), 3.12(\mathrm{~d}, J=7.6 \mathrm{~Hz}, 2 \mathrm{H}), 2.45-2.30(\mathrm{~m}$, $1 \mathrm{H}), 1.17(\mathrm{~d}, J=7.6 \mathrm{~Hz}, 6 \mathrm{H})$.

${ }^{13} \mathrm{C}$ NMR $\left(100 \mathrm{MHz}, \mathrm{CDCl}_{3}\right) \delta(\mathrm{ppm}) 169.5,148.6,130.5,129.7,128.7,128.2,127.3(\mathrm{~m}), 127.2,126.6$, 126.3, 125.8, 124.9, 123.6, 123.0, 43.2, 30.0, 22.6.

Elemental analysis: calcd (\%) for $\mathrm{C}_{19} \mathrm{H}_{17} \mathrm{NS}$ (291.41): C 78.31, H 5.88; found: C 78.61, H 5.74.

\section{1-(Acenaphtho[1,2-b]thiophen-8-yl)ethan-1-one $(4 \mathrm{i})^{[20]}$}

Following the procedure $\mathbf{C}$, from 1-(4-bromo-5-(naphthalen-1-yl)thiophen-2-yl)ethan-1-one $\mathbf{2 i}$ (0.166 g, $0.5 \mathrm{mmol})$, compound $4 \mathbf{i}$ was obtained in $53 \%$ yield $(0.066 \mathrm{~g})$ as a yellow solid: $\mathrm{mp} 149-151^{\circ} \mathrm{C}$. 
${ }^{1} \mathrm{H}$ NMR $\left(400 \mathrm{MHz}, \mathrm{CDCl}_{3}\right) \delta(\mathrm{ppm}) 7.96(\mathrm{~s}, 1 \mathrm{H}), 7.88(\mathrm{~d}, J=7.5 \mathrm{~Hz}, 1 \mathrm{H}), 7.84(\mathrm{~d}, J=7.6 \mathrm{~Hz}, 1 \mathrm{H}), 7.81$ $(\mathrm{d}, J=8.2 \mathrm{~Hz}, 1 \mathrm{H}), 7.76(\mathrm{~d}, J=6.9 \mathrm{~Hz}, 1 \mathrm{H}), 7.63-7.57(\mathrm{~m}, 2 \mathrm{H}), 2.65(\mathrm{~s}, 3 \mathrm{H})$.

${ }^{13} \mathrm{C}$ NMR $\left(100 \mathrm{MHz}, \mathrm{CDCl}_{3}\right) \delta(\mathrm{ppm}) 190.9,149.0,146.9,145.3,134.0,132.9,132.4,129.6,128.3$, $127.9(\mathrm{~m}), 126.7,125.0,122.3,121.2,26.7$.

\section{Acenaphtho[1,2-b]thiophen-8-yl(cyclopropyl)methanone (4k)}

Following the procedure $\mathbf{C}$, from (4-bromo-5-(naphthalen-1-yl)thiophen-2-yl)(cyclopropyl)methanone 2k $(0.179 \mathrm{~g}, 0.5 \mathrm{mmol})$, compound $\mathbf{4 k}$ was obtained in $51 \%$ yield $(0.070 \mathrm{~g})$ as a yellow solid: $\mathrm{mp} 99$ $101^{\circ} \mathrm{C}$.

${ }^{1} \mathrm{H}$ NMR $\left(400 \mathrm{MHz}, \mathrm{CDCl}_{3}\right) \delta(\mathrm{ppm}) 8.07(\mathrm{~s}, 1 \mathrm{H}), 7.88-7.75(\mathrm{~m}, 3 \mathrm{H}), 7.73(\mathrm{~d}, J=6.9 \mathrm{~Hz}, 1 \mathrm{H}), 7.61-7.55$ $(\mathrm{m}, 2 \mathrm{H}), 2.66-2.55(\mathrm{~m}, 1 \mathrm{H}), 1.38-1.29(\mathrm{~m}, 2 \mathrm{H}), 1.14-1.05(\mathrm{~m}, 2 \mathrm{H})$.

${ }^{13} \mathrm{C}$ NMR $\left(100 \mathrm{MHz}, \mathrm{CDCl}_{3}\right) \delta(\mathrm{ppm}) 193.1,148.5,147.5,134.0,133.0,132.5,129.5,128.2,127.9(\mathrm{~m})$, $126.7,124.1,122.2,121.1,18.0,11.4$.

Elemental analysis: calcd (\%) for $\mathrm{C}_{18} \mathrm{H}_{12} \mathrm{OS}$ (276.35): C 78.23, H 4.38; found: C 77.98, H 4.25.

\section{Ethyl acenaphtho[1,2-b]thiophene-8-carboxylate $(4 I)^{[20]}$}

Following the procedure $\mathbf{C}$, from ethyl 4-bromo-5-(naphthalen-1-yl)thiophene-2-carboxylate $2 \mathbf{l}(0.181 \mathrm{~g}$, $0.5 \mathrm{mmol})$, compound $4 \mathrm{I}$ was obtained in $38 \%$ yield $(0.053 \mathrm{~g})$ as a yellow solid: $\mathrm{mp} 178-180{ }^{\circ} \mathrm{C}$.

${ }^{1} \mathrm{H}$ NMR $\left(400 \mathrm{MHz}, \mathrm{CDCl}_{3}\right) \delta(\mathrm{ppm}) 8.12(\mathrm{~s}, 1 \mathrm{H}), 7.90-7.76(\mathrm{~m}, 4 \mathrm{H}), 7.64-7.58(\mathrm{~m}, 2 \mathrm{H}), 4.44(\mathrm{q}, J=7.6$ $\mathrm{Hz}, 2 \mathrm{H}), 1.46(\mathrm{t}, J=7.6 \mathrm{~Hz}, 3 \mathrm{H})$.

\section{8-Isobutylacenaphtho[1,2- $d]$ thiazole $(4 \mathrm{~m})$}

Following the procedure $\mathbf{C}$, from 4-bromo-2-isobutyl-5-(naphthalen-1-yl)thiazole $2 \mathrm{~m}(0.173 \mathrm{~g}, 0.5$ $\mathrm{mmol})$, compound $4 \mathrm{~m}$ was obtained in $21 \%$ yield $(0.028 \mathrm{~g})$ as a yellow oil.

${ }^{1} \mathrm{H}$ NMR $\left(400 \mathrm{MHz}, \mathrm{CDCl}_{3}\right) \delta(\mathrm{ppm}) 8.02(\mathrm{~d}, J=6.9 \mathrm{~Hz}, 1 \mathrm{H}), 7.81(\mathrm{~d}, J=8.1 \mathrm{~Hz}, 2 \mathrm{H}), 7.71(\mathrm{~d}, J=6.9$ $\mathrm{Hz}, 1 \mathrm{H}), 7.61(\mathrm{t}, J=8.1 \mathrm{~Hz}, 1 \mathrm{H}), 7.57(\mathrm{t}, J=8.1 \mathrm{~Hz}, 1 \mathrm{H}), 3.04(\mathrm{~d}, J=7.6 \mathrm{~Hz}, 2 \mathrm{H}), 2.33-2.17(\mathrm{~m}, 1 \mathrm{H})$, $1.10(\mathrm{~d}, J=7.6 \mathrm{~Hz}, 6 \mathrm{H})$.

${ }^{13} \mathrm{C}$ NMR $\left(100 \mathrm{MHz}, \mathrm{CDCl}_{3}\right) \delta(\mathrm{ppm}) 173.6,160.4,133.6,131.9,131.8,130.9,129.2,127.8,127.6$, 127.3, 127.2, 121.8, 121.4, 43.4, 30.1, 22.4.

Elemental analysis: calcd (\%) for $\mathrm{C}_{17} \mathrm{H}_{15} \mathrm{NS}$ (265.37): C 76.94, H 5.70; found: C 77.20, H 5.60.

\section{Acknowledgements}

We are grateful to CNRS, Rennes Metropole and Scientific Ministry of Higher Education and Research of Tunisia for providing financial support.

Keywords: Palladium $\cdot \mathrm{C}-\mathrm{H}$ bond activation $\cdot$ heteroaromatics $\bullet \pi$-extended aromatics $\bullet$ phenanthro[9,10- $b]$ thiophenes 
[1] a) J. E. Anthony, Chem. Rev. 2006, 106, 5028-5048; b) S. Allard, M. Forster, B. Souharce, H. Thiem, U. Scherf, Angew. Chem. Int. Ed. 2008, 47, 4070-4098; c) H. Zhang, D. Wu, S. Hua Liu, J. Yin, Curr. Org. Chem. 2012, 16, 2124-2158; d) C. Wang, H. Dong, W. Hu, Y. Liu, D. Zhu, Chem. Rev. 2012, 112, 2208-2267.

[2] a) C.-L. Sun, B.-J. Li, Z.-J. Shi, Chem. Commun. 2010, 46, 677-685; b) N. Kuhl, M. N. Hopkinson, J. Wencel-Delord, F. Glorius, Angew. Chem. Int. Ed. 2012, 51, 10236-10254; c) B.-J. Li, Z.-J. Shi, Chem. Soc. Rev. 2012, 41, 5588-5598; d) J. Wencel-Delord, F. Glorius, Nature Chem. 2013, 5, 369-375; e) S. I. Kozhushkov, L. Ackermann, Chem. Sci. 2013, 4, 886-896; f) I. Kuzhushkov, H. K. Potukuchi, L. Ackermann, Catal. Sci. Technol. 2013, 3, 562-571; g) R. Rossi, F. Bellina, M. Lessi, C. Manzini, Adv. Synth. Catal. 2014, 356, 17-117; h) L. Theveau, C. Schneider, C. Fruit, C. Hoarau, ChemCatChem 2016, 8, 3183-3194; i) R. Rossi, M. Lessi, C. Manzini, G. Marianetti, F. Bellina, Tetrahedron 2016, 72, 1795-1837; j) X. Shi, A. Sasmal, J.-F. Soulé, H. Doucet, Chem. Asian J. 2018, 13, 143-157; j) S. Mao, H. Li, X. Shi, J.-F. Soule, H. Doucet, ChemCatChem, 2019, 11, 269-286.

[3] a) Y. Akita, A. Inoue, K. Yamamoto, A. Ohta, T. Kurihara, M. Shimizu, Heterocycles 1985, 23, 2327-2333; b) A. Ohta, Y. Akita, T. Ohkuwa, M. Chiba, R. Fukunaga, A. Miyafuji, T. Nakata, N. Tani, Y. Aoyagi, Heterocycles 1990, 31, 1951-1958.

[4] a) B. Liegault, D. Lapointe, L. Caron, A. Vlassova, K. Fagnou, J. Org. Chem. 2009, 74, 1826-1834; b) D. Roy, S. Mom, D. Lucas, H. Cattey, J.-C. Hierso, H. Doucet, Chem. Eur. J. 2011, 17, 64536461.

[5] Synthesis of PHHs containing fully unsaturated 6-membered rings fused with a thiophene core: a) X. H. Cheng, S.-S. Jester, S. Höger, Macromolecules 2004, 37, 7065-7068; b) K. Saito, P. K. Chikkade, M. Kanai, Y. Kuninobu, Chem. Eur. J. 2015, 21, 8365-8368; c) W. Hagui, N. Besbes, E. Srasra, T. Roisnel, J.-F. Soulé, H. Doucet, Org. Lett. 2016, 18, 4182-4185; d) W. Matsuoka, H. Ito, K. Itami, Angew. Chem. Int. Ed. 2017, 56, 12224-12228; e) K. Ozaki, W. Matsuoka, H. Ito, K. Itami, Org. Lett. 2017, 19, 1930-1933; f) X. Gu, H. Li, B. Shan, Z. Liu, Q. Miao, Org. Lett.,2017, 19, 22462249.

[6] Synthesis of PHHs containing fully unsaturated 6-membered rings fused with a thiazole core: $\mathrm{X}$. Shi, J.-F. Soulé, H. Doucet, J. Org. Chem. 2017, 82, 3886-3894.

[7] Synthesis of PHHs containing fully unsaturated 6-membered rings fused with a benzofuran core: a) Z. Liu, X. Zhang, R. C. Larock, J. Am. Chem. Soc. 2005, 127, 15716-15717; b) W. C. Fu, Z. Wang, W. T. K. Chan, Z. Lin, F. Y. Kwong, Angew. Chem. Int. Ed. 2017, 56, 7166-7170; see also $5 b$ and 5e; fused with a furan core: c) B. Lu, J. Wu, N. Yoshikai, J. Am. Chem. Soc. 2014, 136, 11598-11601.

[8] Synthesis of PHHs containing fully unsaturated 5-membered rings fused with thiophene or furan cores: a) M. Smet, J. Van Dijk, W. Dehaen, Synlett 1999, 495-497; b) A. R. Mohebbi, F. Wudl, Chem. Eur. J. 2011, 17, 2642-2646.

[9] Synthesis of PHHs containing fully unsaturated 6-membered rings fused with pyrrole or indole cores: a) J. Gao, Y. Shao, J. Zhu, J. Zhu, H. Mao, X. Wang, X. Lv, J. Org. Chem. 2014, 79, 90009008; b) J. H. Kim, S. Y. Choi, J. Bouffard, S.-G. Lee, J. Org. Chem. 2014, 79, 9253-9261; c) S. Ito, Y. Tokimaru, K. Nozaki, Angew. Chem. Int. Ed. 2015, 54, 7256-7260; d) H. Yokoi, Y. Hiraoka, S. Hiroto, D. Sakamaki, S. Seki, H. Shinokubo, Nat. Commun. 2015, 6, 8215; e) S. K. Bhunia, A. Polley, R. Natarajan, R. Jana, Chem. Eur. J. 2015, 21, 16786-16791; f) H. Kitano, W. Matsuoka, H. Ito, K. Itami, Chem. Sci. 2018, 9, 7556-7561.

[10] L. Zhao, C. Bruneau, H. Doucet, ChemCatChem 2013, 5, 255-262.

[11] J. Roger, H. Doucet, Tetrahedron 2009, 65, 9772-9781. 
[12] a) C. Zheng, S. Pu, J. Xu, M. Luo, D. Huang, L. Shen, Tetrahedron 2007, 63, 5437-5449; b) H. Zhang, J. Fan, Z. lqbal, D.-B. Kuang, L. Wang, D. Cao, H. Meier, Dyes Pigm. 2013, 99, 74-81; c) P. J. Milner, Y. Yang, S. L. Buchwald, Organometallics 2015, 34, 4775-4780.

[13] a) K. L. Milkiewicz, D. J. Parks, T. Lu, Tetrahedron Lett. 2003, 44, 4257-4260; b) X. Tian, L. Song, M. Rudolph, Q. Wang, X. Song, F. Rominger, A. S. K. Hashmi, Org. Lett. 2019, 21, 1598-1601.

[14] M. Giraud, A. Leaustic, R. Guillot, P. Yu, P. G. Lacroix, K. Nakatani, R. Pansu, F. Maurel, J. Mater. Chem. 2007, 17, 4414-4425.

[15] a) P. Cagniant, G. Kirsch, C. R. Hebd. Séances Acad. Sci. C 1975, 281, 393-395; b) G. K. Kar, B. G. Chatterjee, J. K. Ray, Org. Prep. Proced. Int. 1988, 20, 213-222.

[16] D. D. Chapman, J. K. Elwood, D. W. Heseltine, H. M. Hess, D. W. Kurtz, J. Org. Chem. 1977, 42, 2474-2480.

[17] T. Cantat, E. Génin, C. Giroud, G. Meyer, A. Jutand, J. Organomet. Chem. 2003, 687, 365-376.

[18] W. Susanto, C.-Y. Chu, W. J. Ang, T.-C. Chou, L.-C. Lo, Y. Lam, J. Org. Chem. 2012, 77, 27292742.

[19] T. T. Dang, M. Bonneau, J. A. G. Williams, H. Le Bozec, H. Doucet, V. Guerchais, Eur. J. Inorg. Chem. 2015, 2956-2964.

[20] S. Hauptmann, M. Scholz, H. J. Koehler, H. J. Hofmann, J. Prak. Chem. 1969, 311, 614-620. 